\title{
Dppm-derived phosphonium salts and ylides as ligand precursors for s-block organometallics
}

\author{
Jens Langer,* Sascha Meyer, Feyza Dündar, Björn Schowtka, Helmar Görls, \\ and Matthias Westerhausen \\ Institute of Inorganic and Analytical Chemistry, Friedrich-Schiller-University Jena \\ Humboldtstraße 8, D-07743 Jena, Germany \\ E-mail:j.langer@uni-jena.de
}

\section{Dedicated to Professor Rainer Beckert on the Occasion of his $60^{\text {th }}$ Birthday}

\begin{abstract}
The addition reaction of 1,1-bis(diphenylphosphino)methane (dppm) and haloalkanes R-X yields the corresponding phosphonium salts $\left[\mathrm{Ph}_{2} \mathrm{PCH}_{2} \mathrm{PPh}_{2} \mathrm{R}\right] \mathrm{X}(\mathbf{1 a}: \mathrm{R}=\mathrm{Me}, \mathrm{X}=\mathrm{I} ; \mathbf{1 b}: \mathrm{R}=\mathrm{Et}, \mathrm{X}=\mathrm{Br}$; 1c: $\mathrm{R}=i \operatorname{Pr}, \mathrm{X}=\mathrm{I}$; 1d: $\mathrm{R}=\mathrm{CH}_{2} \mathrm{Mes}, \mathrm{X}=\mathrm{Br}$; $1 \mathrm{e}: \mathrm{R}=t \mathrm{Bu}, \mathrm{X}=\mathrm{Br}$ ). In case of the synthesis of $1 \mathbf{e}$, $\left[\mathrm{Ph}_{2} \mathrm{MePH}\right] \mathrm{Br}(\mathbf{3})$ was identified as a by-product. Deprotonation of $\mathbf{1}$ by $\mathrm{KO} t \mathrm{Bu}$ offers access to the corresponding phosphonium ylides $\left[\mathrm{Ph}_{2} \mathrm{PCHPPh}_{2} \mathrm{R}\right](\mathbf{2 a}: \mathrm{R}=\mathrm{Me} ; \mathbf{2 b}: \mathrm{R}=\mathrm{Et} ; \mathbf{2} \mathbf{c}: \mathrm{R}=i \operatorname{Pr}$; 2d: $\mathrm{R}=\mathrm{CH}_{2} \mathrm{Mes}$ ) in good yields. Further deprotonation of $\mathbf{2 a}$ using $n$-butyllithium allows the isolation of the lithium complex $\left[\mathrm{Li}\left(\mathrm{Ph}_{2} \mathrm{PCHPPh}_{2} \mathrm{CH}_{2}\right)\right]_{\mathrm{n}}(4)$ and its monomeric tmeda adduct [(tmeda) $\left.\mathrm{Li}\left(\mathrm{Ph}_{2} \mathrm{PCHPPh}_{2} \mathrm{CH}_{2}\right)\right]$ (4a). All compounds were characterized by NMR measurements and, except of $\mathbf{4}$, by X-ray diffraction experiments.
\end{abstract}

Keywords: Phosphonium salt, phosphonium ylide, lithium, lithium phosphorus coupling

\section{Introduction}

Phosphonium ylides gained tremendous importance in organic chemistry, since Wittig and coworkers developed their alkene synthesis in the early 50's. ${ }^{1}$ Nowadays, the Wittig reaction is text book chemistry ${ }^{2}$ and numerous applications and variations of this reaction are known. ${ }^{3}$ Beside their impact on synthetic organic chemistry, the bonding situation of phosphonium ylides is a subject of considerable interest; for instance the (non-existing) d-orbital participation and hypervalence have been studied intensively. ${ }^{4}$

In inorganic chemistry, neutral phosphonium ylides and their deprotonated anions are interesting ligands for s-block and d-block metals. ${ }^{5}$ Especially ylides derived from bidentate diphosphanes proved to be useful in early studies due to the additional chelate effect. ${ }^{6}$ So far 
predominately keto stabilized ylides were used as neutral ligands ${ }^{7}$ and a variety of coordination modes of these derivatives with different d-block metals was observed.

In contrast, the s-block organometallic chemistry of this type of chelating ylide ligands and their anions is still underdeveloped. Continuing our previous investigation of the coordination chemistry of anionic ligands derived from 1,1-bis(diphenylphosphino)methane (dppm), ${ }^{8}$ the synthesis of a series of phosphonium salts and their phosphonium ylides is presented.

\section{Results and Discussion}

Chelating ylidic ligands are easily obtainable from 1,1-bis(diphenylphosphino)methane (dppm) by well known synthetic protocols. The stepwise deprotonation of phosphonium salts obtained from dppm and simple alkyl halides have been previously used by e.g. the groups of Issleib ${ }^{9}$ and Schmidbaur. ${ }^{10}$

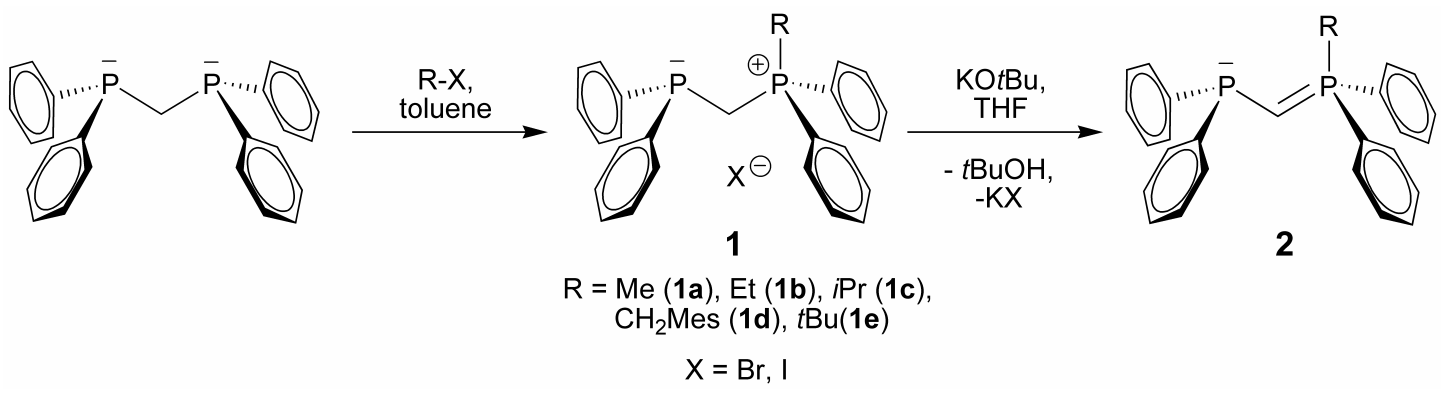

Scheme 1. Synthesis of 1 and 2.

Applying this approach, a series of phosphonium salts was synthesized in order to test its limitations (see scheme 1). Table 1 summarizes the reaction conditions and yields.

Table 1. Synthesis of 1a-e

\begin{tabular}{lcccccc}
\hline Compound & $\mathrm{R}-\mathrm{X}$ & $\begin{array}{c}\text { Equivalents } \\
\mathrm{R}-\mathrm{X}\end{array}$ & $\begin{array}{c}\mathrm{T} \\
{\left[{ }^{\circ} \mathrm{C}\right]}\end{array}$ & $\begin{array}{c}\text { Reaction } \\
\text { time [d] }\end{array}$ & $\begin{array}{c}\text { Isolated } \\
\text { Yield [\%] }\end{array}$ & Reference \\
\hline $\mathbf{1 a}$ & $\mathrm{MeI}$ & 1 & 80 & 0.17 & 97.5 & {$[10 \mathrm{~b}]$} \\
$\mathbf{1 b}$ & $\mathrm{EtBr}$ & 6.1 & 50 & 7 & 51 & - \\
$\mathbf{1 c}$ & $i \mathrm{PrI}$ & 4.1 & 80 & 7 & 37 & - \\
$\mathbf{1 d}$ & $\mathrm{MesCH} \mathrm{CH}_{2} \mathrm{Br}$ & 1 & 80 & 2 & 99 & - \\
$\mathbf{1 e}$ & $t \mathrm{BuBr}$ & 4.3 & 70 & 21 & 19 & - \\
\hline
\end{tabular}

While iodomethane gave the corresponding derivative $\left[\mathrm{Ph}_{2} \mathrm{PCH}_{2} \mathrm{PPh}_{2} \mathrm{CH}_{3}\right] \mathrm{I}(\mathbf{1 a})$ in excellent yield after short reaction time as also known from the literature, ${ }^{10 \mathrm{~b}}$ other substrates required more forcing reaction conditions. An increase of the bulkiness of the alkyl group $\mathrm{R}$ in primary 
haloalkanes $\mathrm{R}-\mathrm{CH}_{2}-\mathrm{X}$ from $\mathrm{R}=\mathrm{H}$ to $\mathrm{R}=\mathrm{CH}_{3}$ and further to $\mathrm{R}=t \mathrm{Bu}$ results in longer reaction times and lower yields in case of $\left[\mathrm{Ph}_{2} \mathrm{PCH}_{2} \mathrm{PPh}_{2} \mathrm{CH}_{2} \mathrm{CH}_{3}\right] \mathrm{Br}(\mathbf{1 b})$ and no transformation at all in case of neopentyl bromide as substrate. This significantly reduced reactivity is in agreement with earlier studies of $\mathrm{S}_{\mathrm{N}} 2$ reactions of neopentyl systems, which are often accompanied by rearrangement to the corresponding 2-methyl-2-butyl derivatives. ${ }^{11}$

As expected for $\mathrm{S}_{\mathrm{N}} 2$ reactions, longer reaction times and an excess of substrate were also necessary in case of secondary haloalkanes such as 2-iodopropane to obtain the desired product. A yield of only $37 \%$ of $\left[\mathrm{Ph}_{2} \mathrm{PCH}_{2} \mathrm{PPh}_{2} \mathrm{CH}\left(\mathrm{CH}_{3}\right)_{2}\right] \mathrm{I}$ (1c) after one week is far from satisfying but the straightforward product isolation just by filtration and the easy recovery of unreacted dppm by removing all volatiles of the mother liquor partially compensate this downside.

Prolonged reaction times of three weeks make even the $t$-butyl derivative $\left[\mathrm{Ph}_{2} \mathrm{PCH}_{2} \mathrm{PPh}_{2} \mathrm{C}\left(\mathrm{CH}_{3}\right)_{3}\right] \mathrm{Br}$ (1e) accessible, but the yields remained low (19\%). Even longer reaction times resulted in additional product formation, but also led to the formation of byproducts, which may arise from $\mathrm{O}_{2}$ or $\mathrm{H}_{2} \mathrm{O}$ leaking into the reaction flask during these extremely long reaction times. One by-product, namely $\left[\mathrm{Ph}_{2} \mathrm{MePH}\right]^{+} \mathrm{Br}^{-}(3)$, was identified by X-ray diffraction experiments.

In case of benzyl derivatives, excellent yields were achieved as demonstrated for the (2,4,6-trimethylphenyl)methyl derivative $\left[\mathrm{Ph}_{2} \mathrm{PCH}_{2} \mathrm{PPh}_{2} \mathrm{CH}_{2} \mathrm{Mes}\right] \mathrm{Br}$ (1d). The corresponding chloride derivative gives the same yield under identical reaction conditions. ${ }^{10 \mathrm{a}}$ Tables 2 and 3 summarize selected NMR data and structural parameters of the isolated compounds 1a-e. The molecular structures of $\mathbf{1 c}$ and $\mathbf{1 d}$ are shown in Figure 1 as representative examples.

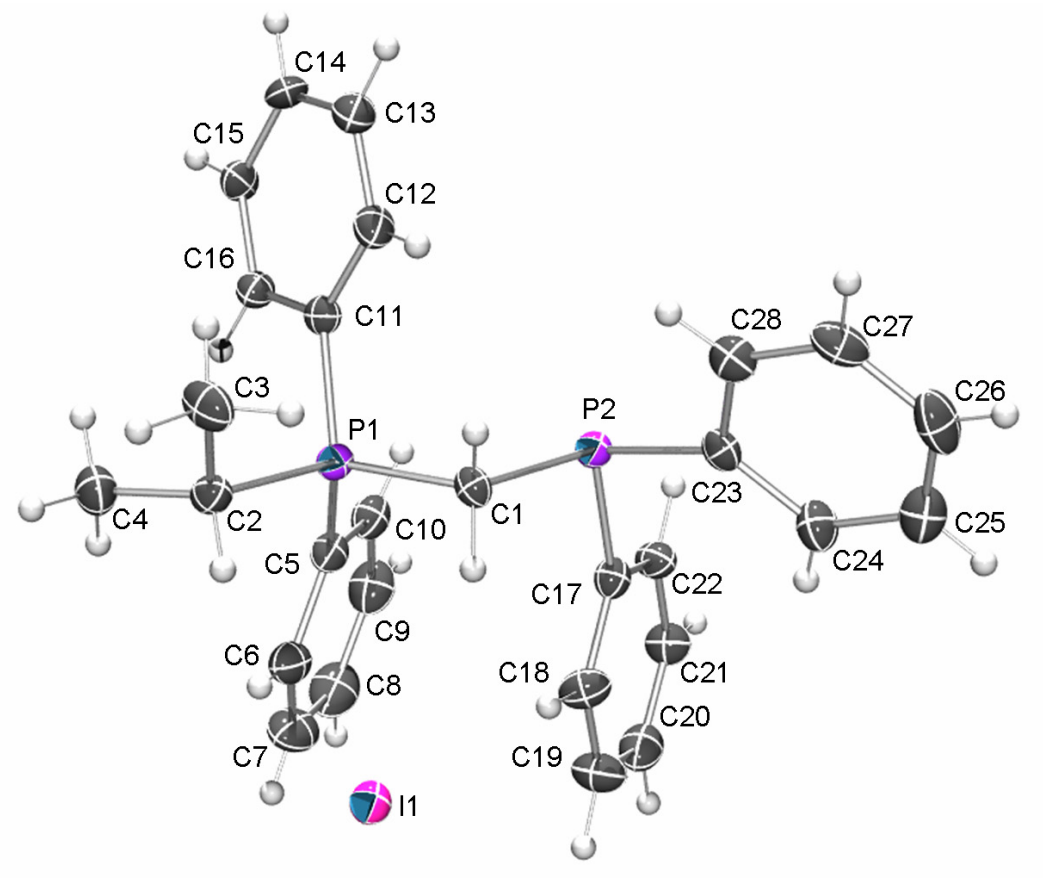




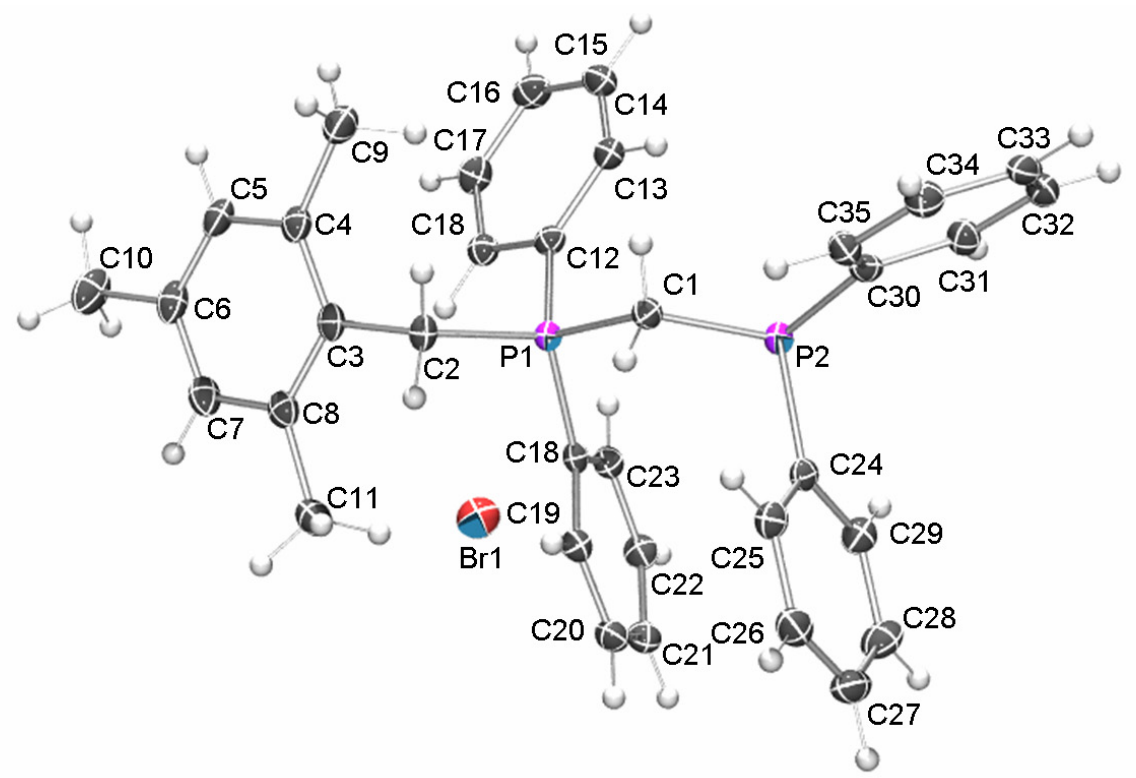

Figure 1. Molecular structures and numbering schemes of 1c (top) and 1d (bottom). Cocrystallized $\mathrm{CHCl}_{3}$ in $\mathbf{1 c}$ is omitted for clarity. The ellipsoids represent a probability of $50 \%$, the $\mathrm{H}$ atoms are shown with arbitrary radii.

Table 2. Selected bond lengths and angles of 1, 2 and 4

\begin{tabular}{|c|c|c|c|c|c|c|}
\hline \multirow[t]{2}{*}{ Entry } & \multicolumn{5}{|c|}{ Bond lengths $[\AA ̊]$} & \multirow{2}{*}{$\frac{\text { Angle }\left[{ }^{\circ}\right]}{\mathrm{P}^{\mathrm{III}}-\mathrm{C} 1-\mathrm{P}^{\mathrm{V}}}$} \\
\hline & $\mathrm{P}^{\mathrm{III}}-\mathrm{C} 1$ & $\mathrm{P}^{\mathrm{III}}-\mathrm{C}(\mathrm{Ph})^{\mathrm{a}}$ & $\mathrm{P}^{\mathrm{V}}-\mathrm{C} 1$ & $\mathrm{P}^{\mathrm{V}}-\mathrm{C} 2$ & $\mathrm{P}^{\mathrm{V}}-\mathrm{C}(\mathrm{Ph})^{\mathrm{a}}$ & \\
\hline \multicolumn{7}{|c|}{ Phosphonium salts } \\
\hline $1 a^{b}$ & $\mathrm{c}$ & $\mathrm{c}$ & $1.782(5)$ & $1.802(5)$ & $1.792(5)$ & $\mathrm{c}$ \\
\hline $1 b^{d}$ & $1.861(2)$ & $1.832(2)$ & $1.796(2)$ & $1.804(2)$ & $1.793(2)$ & $114.69(11)$ \\
\hline 1c & $1.872(5)$ & $1.831(5)$ & $1.810(5)$ & $1.827(5)$ & $1.798(5)$ & $110.7(2)$ \\
\hline 1d & $1.867(2)$ & $1.838(2)$ & $1.797(2)$ & $1.819(2)$ & $1.792(2)$ & $111.48(10)$ \\
\hline 1e & $1.889(3)$ & $1.834(3)$ & $1.803(3)$ & $1.859(3)$ & $1.801(3)$ & $111.41(17)$ \\
\hline \multicolumn{7}{|c|}{ Ylides } \\
\hline $2 \mathbf{a}$ & $1.767(3)$ & $1.845(3)$ & $1.687(3)$ & $1.812(4)$ & $1.815(3)$ & $119.64(19)$ \\
\hline $2 b$ & $1.7642(14)$ & $1.8438(14)$ & $1.6866(14)$ & $1.8267(15)$ & $1.8178(14)$ & $120.25(8)$ \\
\hline 2c & $1.764(3)$ & $1.846(3)$ & $1.690(3)$ & $1.824(3)$ & $1.818(3)$ & $121.23(16)$ \\
\hline 2d & $1.773(2)$ & $1.842(2)$ & $1.700(2)$ & $1.848(2)$ & $1.818(2)$ & $117.79(13)$ \\
\hline \multicolumn{7}{|c|}{ Lithium complex } \\
\hline $\mathbf{4 a}$ & $1.753(3)$ & $1.838(3)$ & $1.696(3)$ & $1.719(3)$ & $1.839(3)$ & $117.71(15)$ \\
\hline
\end{tabular}

${ }^{a}$ Average value. ${ }^{b}$ Molecule A of two independent molecules. ${ }^{c}$ No accurate values are available due to a disorder of the $-\mathrm{CH}_{2} \mathrm{PPh}_{2}$ group of the molecule. ${ }^{\mathrm{d}}$ Data of $\mathbf{1 b}$ toluene was used. 
Table 3. Selected NMR data of compounds 1, 2 and 4

\begin{tabular}{|c|c|c|c|c|c|c|c|}
\hline \multirow[t]{2}{*}{ Entry } & \multicolumn{2}{|c|}{${ }^{1} \mathrm{H}$ NMR } & \multicolumn{2}{|c|}{${ }^{13} \mathrm{C}$ NMR } & \multicolumn{3}{|c|}{${ }^{31} \mathrm{P} N M R$} \\
\hline & $\delta \mathrm{P}_{-} \mathrm{CH}_{\mathrm{x}}-\mathrm{P}$ & ${ }^{2} J_{\mathrm{H}, \mathrm{P}}[\mathrm{Hz}]$ & $\delta \mathrm{P}-\mathrm{CH}_{\mathrm{x}}-\mathrm{P}$ & ${ }^{1} J_{\mathrm{C}, \mathrm{P}}[\mathrm{Hz}]$ & $\delta \mathrm{P}^{\mathrm{III}}$ & $\delta \mathrm{P}^{\mathrm{V}}$ & ${ }^{2} J_{\mathrm{P}, \mathrm{P}}[\mathrm{Hz}]$ \\
\hline \multicolumn{8}{|c|}{ Phosphonium salts } \\
\hline 1a & 4.12 & 14.7 & 22.5 & $51.2 ; 34.7$ & -22.3 & 26.7 & 59.7 \\
\hline $1 \mathrm{~b}$ & 4.20 & 14.3 & 20.2 & $49.8 ; 34.1$ & -23.2 & 33.8 & 58.2 \\
\hline 1c & 4.05 & 13.0 & 18.7 & $48.7 ; 33.3$ & -24.6 & 38.1 & 58.7 \\
\hline 1d & 4.45 & 13.8 & 22.0 & $47.7 ; 32.9$ & -23.8 & 25.8 & 61.3 \\
\hline 1e & 3.95 & 12.3 & 18.4 & $46.2 ; 35.7$ & -23.0 & 41.2 & 60.5 \\
\hline \multicolumn{8}{|c|}{ Ylides } \\
\hline 2a & 1.23 & $11.4 ; 1.6$ & 8.5 & $118.9 ; 11.2$ & -16.3 & 19.4 & 158.3 \\
\hline $2 \mathbf{b}$ & a & a & 3.9 & $117.3 ; 10.6$ & -16.3 & 27.7 & 150.7 \\
\hline $2 \mathrm{c}$ & 1.10 & $\sim 10 ; 1.3$ & 0.0 & $116.2 ; 10.7$ & -15.7 & 33.7 & 135.7 \\
\hline 2d & 1.06 & $11.2 ; 2.7$ & 10.5 & $117.0 ; 10.3$ & -15.8 & 24.2 & 150.6 \\
\hline \multicolumn{8}{|c|}{ Lithium complex } \\
\hline $4 \mathbf{a}$ & 1.12 & $11.0 ; 8.9$ & 14.5 & $135.0 ; 19.2$ & -13.5 & 36.4 & 139.3 \\
\hline
\end{tabular}

${ }^{a}$ Accurate value is not available due to overlapping signals.

Phosphonium salts of the type $\mathbf{1}$ can easily be deprotonated by strong bases such as $\mathrm{Me}_{3} \mathrm{PCH}_{2}{ }^{10 \mathrm{~b}} \mathrm{NaNH}_{2},{ }^{9}$ or $\mathrm{KO} t \mathrm{Bu}$ to form the corresponding ylides. Depending on the nature of the substituent $\mathrm{R}$, deprotonation either takes place in the bridging $\mathrm{P}-\mathrm{CH}_{2}-\mathrm{P}$ group or in the substituent (see scheme 2).
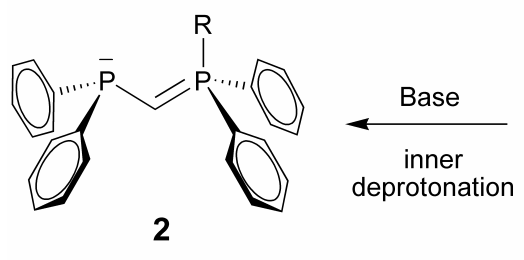

$\mathrm{R}=\mathrm{Me}(\mathbf{2 a}), \mathrm{Et}(\mathbf{2} \mathbf{b})$,

$i \operatorname{Pr}(\mathbf{2 c}), \mathrm{CH}_{2} \operatorname{Mes}(\mathbf{2 d})$
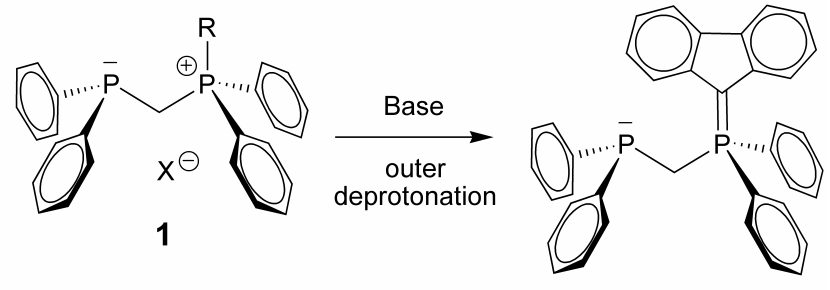

Scheme 2. Substituent dependent regioselective deprotonation of 1 with $\mathrm{R}$ being hydrocarbon groups.

While in substituents containing for instance $\beta$-carbonyl groups the anionic charge of an adjacent ylide is greatly stabilized and consequently deprotonation takes place in the substituent (outer deprotonation), ${ }^{7}$ simple alkyl-substituted phosphonium salts like 1a-c are deprotonated in the bridge (inner deprotonation). ${ }^{9,10 \mathrm{~b}}$ Like for the parent benzyl derivative, ${ }^{9}$ inner deprotonation was found for 1d, indicating that a $\mathrm{Ph}_{2} \mathrm{P}$ group provides superior stabilization for the formed ylide. In contrast, deprotonation within the substituent was reported for the related fluorenyl 
derivative. ${ }^{10 a}$ Beside a simple deprotonation reaction, the rearrangement of in situ generated (1diphenylphosphino-1-methylethyl)methylenediphenylphosphorane offers an alternative strategy to $\left[\mathrm{Ph}_{2} \mathrm{PCHPPh}_{2} \mathrm{CH}\left(\mathrm{CH}_{3}\right)_{2}\right](\mathbf{2 c})$ in low yield. ${ }^{12}$

The phosphonium ylides $\mathbf{2 a - d}$ were isolated as off-white to pale yellow substances and characterized by NMR spectroscopy. Selected NMR data of these compounds is summarized in table 2. Additionally, the molecular structures of all four compounds were determined by X-ray diffraction experiments to elucidate the structural changes accompanying the deprotonation. Figure 2 shows the molecular structures of $\mathbf{2 c}$ and $\mathbf{2 d}$ as typical examples.

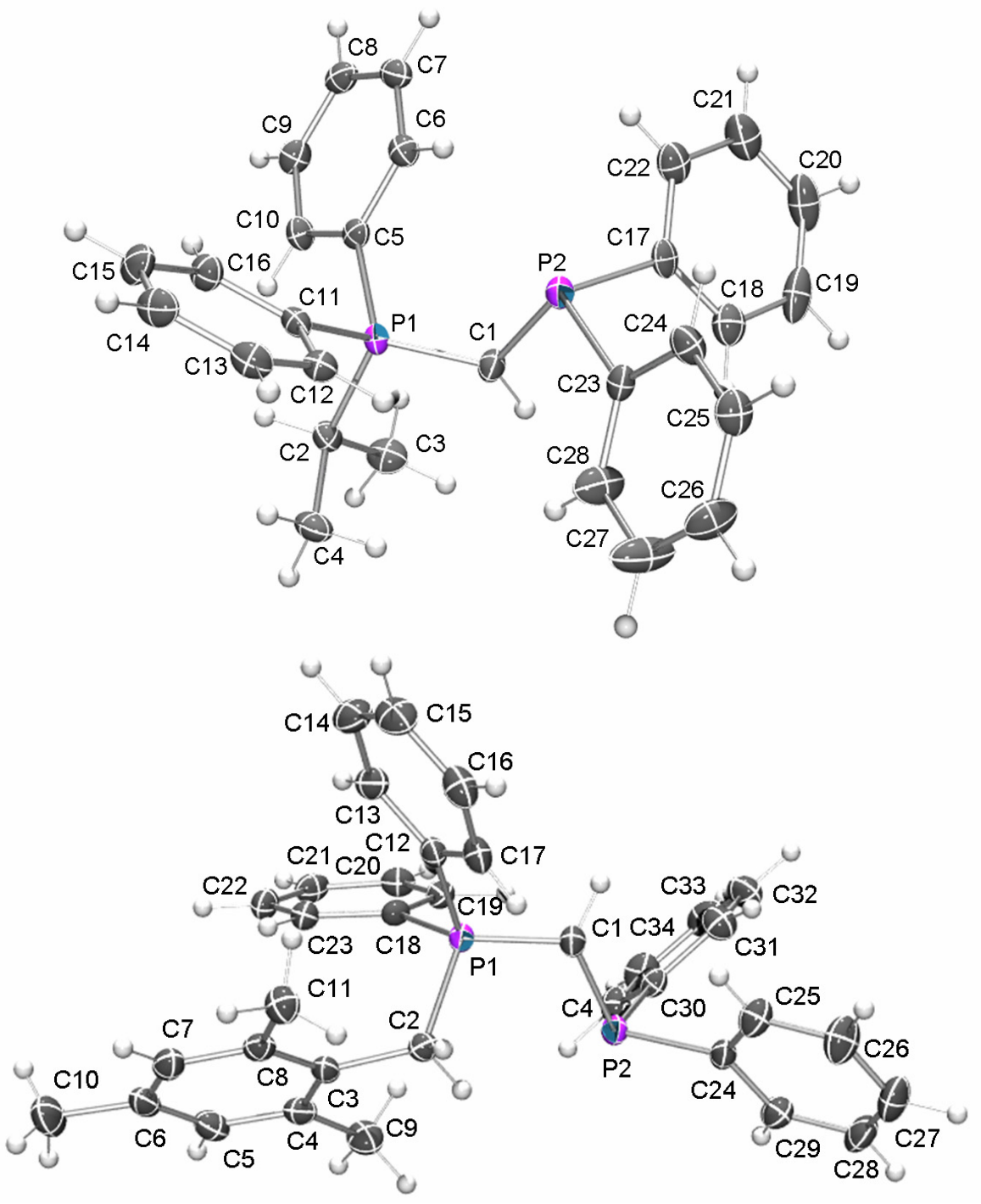

Figure 2. Molecular structures and numbering schemes of 2c (top) and 2d (bottom). Cocrystallized $\mathrm{Et}_{2} \mathrm{O}(\mathbf{2 c})$ is omitted for clarity. The ellipsoids represent a probability of $50 \%$, the $\mathrm{H}$ atoms are shown with arbitrary radii. 
In comparison to the data of the phosphonium salts, shortening of both $\mathrm{P}-\mathrm{C}$ bonds around the ylidic carbon by roughly $0.1 \AA$ was observed, indicating a certain amount of charge delocalization towards the $\mathrm{Ph}_{2} \mathrm{P}$ fragment. The $\mathrm{P}-\mathrm{C}-\mathrm{P}$ angle in all four derivatives is close to $120^{\circ}$ with $\left[\mathrm{Ph}_{2} \mathrm{PCHPPh}_{2} \mathrm{CH}_{2} \mathrm{Mes}\right]$ (2d) showing the largest deviation with $117.79^{\circ}$. In this derivative, some degree of pyramidalization at $\mathrm{C} 1$ was found, resulting in an angle sum of $353.7^{\circ}$.

Aside from the potential use of phosphonium ylides of type 2 in the Wittig reaction ${ }^{13}$ or as mono- or bidentate neutral ligands, they are also easy-to-handle precursors for monoanionic ligands. The deprotonation of $\left[\mathrm{Ph}_{2} \mathrm{PCHPPh}_{2} \mathrm{CH}_{3}\right]$ (2a) at the $\mathrm{CH}_{3}$ group by methyllithium or $n$-butyllithum has been reported earlier ${ }^{10 b, 14}$ and the resulting solutions have been used to transfer the anionic ligand to e.g. yttrium or nickel complexes, ${ }^{14,15}$ but little is known about the intermediately formed lithium compound itself.

This lithium compound can be isolated in pure form, when the deprotonation by $n$ butyllithium is performed in toluene. Due to the lack of additional neutral donor ligands, a polymeric structure of the product has to be assumed making it sparingly soluble in toluene and facilitating the isolation. The obtained very moisture sensitive white powder of $\left[\mathrm{Li}\left(\mathrm{Ph}_{2} \mathrm{PCHPPh}_{2} \mathrm{CH}_{2}\right)\right]_{\mathrm{n}}(4)$ is soluble in donor solvents like diethyl ether, THF or THP. In THF solution the polymer is split into monomeric units probably containing two coordinated thf molecules to fill the coordination sites at lithium. A complete dissociation into a $\left[\mathrm{Li}(\operatorname{thf})_{4}\right]^{+}$ cation and a liberated anionic ligand as observed for related $\left[\mathrm{Li}(\mathrm{dme})_{3}\right]\left[\mathrm{H}_{3} \mathrm{BPPh}_{2} \mathrm{CHPPh}_{2} \mathrm{BH}_{3}\right]$ can safely be excluded. ${ }^{8}$ In the ${ }^{7} \mathrm{Li}\{\mathrm{H}\}$ NMR as well as in the ${ }^{31} \mathrm{P}\{\mathrm{H}\}$ NMR spectrum a ${ }^{1} J_{\mathrm{PLi}}$ coupling of approximately $36 \mathrm{~Hz}$ was observed even at ambient temperature, allowing the description of $\mathbf{4}$ as a strong contact ion pair in THF solution. The observed coupling constant is rather small but falls into the same order of magnitude as observed for other compounds. ${ }^{16}$ For the closely related $\mathrm{Li}\left[\mathrm{Ph}_{2} \mathrm{PCHP}(\mathrm{S}) \mathrm{Ph}_{2}\right]$ in diethyl ether a coupling constant of $54 \mathrm{~Hz}$ was reported. $^{16 \mathrm{c}}$

Recrystallization of 4 from $N, N, N^{\prime}, N^{\prime}$-tetramethylethylendiamine (tmeda) yielded the mononuclear [(tmeda) $\left.\mathrm{Li}\left(\mathrm{Ph}_{2} \mathrm{PCHPPh}_{2} \mathrm{CH}_{2}\right)\right]$ (4a) resembling the bonding situation of the thf solvate. NMR measurement in $\left[\mathrm{D}_{8}\right] \mathrm{THF}$ indicates, that the tmeda ligand can be replaced by THF and identical spectra as in case of $\mathbf{4}$ were observed for $\mathbf{4 a}$ aside from the signals of noncoordinated tmeda. The molecular structure of $\mathbf{4 a}$ is displayed in Figure 3.

The lithium atom is in a distorted tetrahedral coordination sphere surrounded by a phosphorus atom and a carbon atom of the ylidic ligand and the two nitrogen donors of tmeda. The bond lengths within the organometallic five-membered ring indicate electron delocalization and partial multiple bond character throughout the whole CPCP fragment. In comparison to the structurally characterized nickel complex $\left[\mathrm{Ni}\left(\mathrm{Ph}_{2} \mathrm{PCHPPh}_{2} \mathrm{CH}_{2}\right)_{2}\right]^{15}$ and the yttrium complex $\left[\mathrm{Y}\left(\mathrm{Ph}_{2} \mathrm{PCHPPh}_{2} \mathrm{CH}_{2}\right)_{3}\right]{ }^{14}$ containing the same ligand, slight differences become obvious. Especially, an increasing $\mathrm{P}-\mathrm{CH}_{2}$ bond length from lithium (1.719(3) $\AA$ ) to yttrium (1.744(7)$1,751(6) \AA)$ and nickel (1.772(7) $\AA$ ) was found and interpreted as decreasing multiple bond character between these atoms. This observation is in agreement with the assumed increasing $\sigma$ bond character of the metal-carbon bond to the $\mathrm{CH}_{2}$ group in this row, leading to formal $\mathrm{sp}^{3}$ 
hybridization of this carbon atom in the nickel complex, whereas the lithium complex 4 a can be regarded as an ion pair.

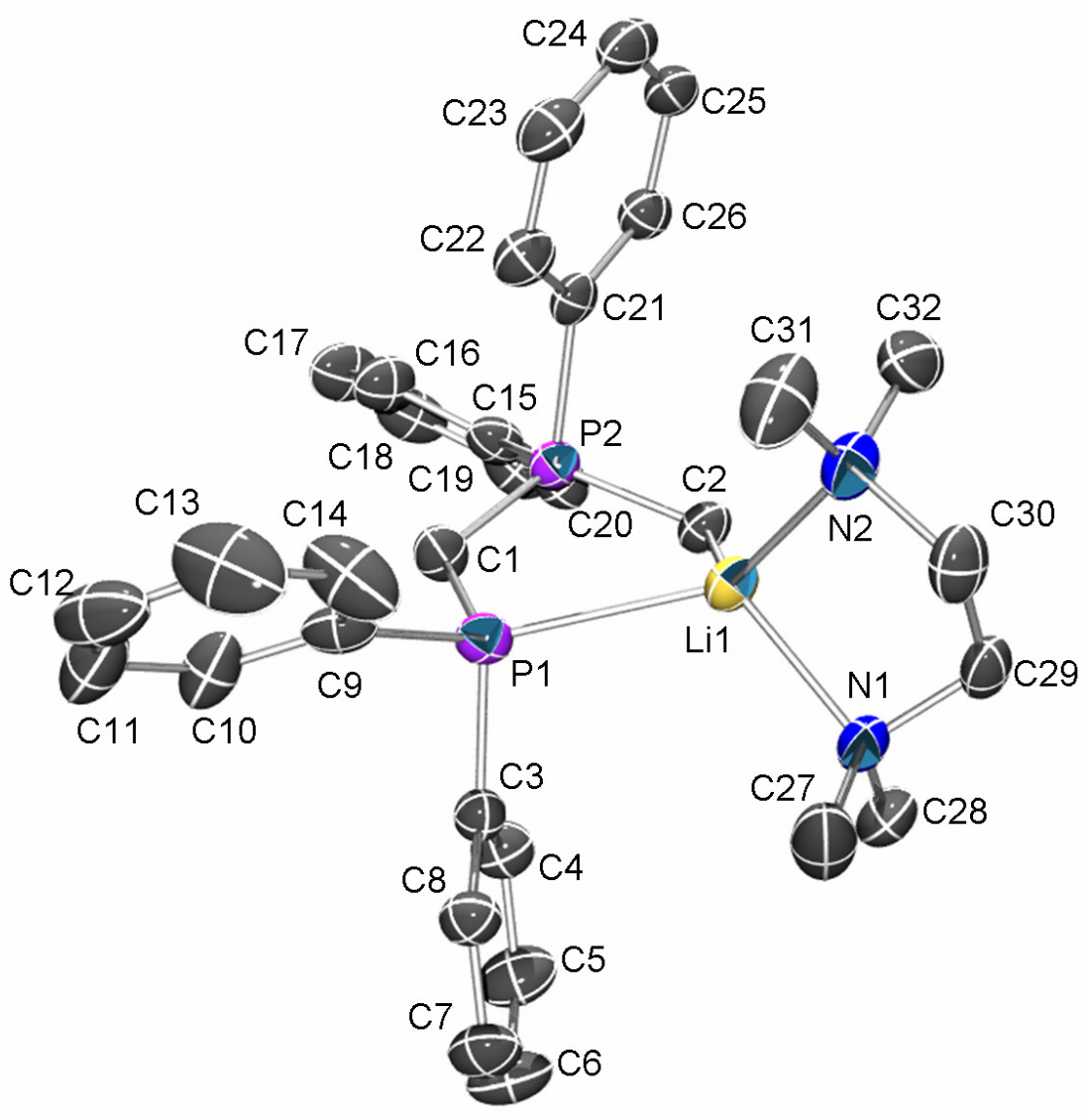

Figure 3. Molecular structure and numbering scheme of 4a. H-atoms and co-crystallized tmeda are omitted for clarity. The ellipsoids represent a probability of 50\%. Selected bond lengths $(\AA)$ and angles (deg): Li1-N1 2.091(5), Li1-N2 2.084(5), Li1-C2 2.161(5), Li1-P1 2.549 (5), N1-Li1N2 88.89(19), N1-Li1-C2 115.7(2), N2-Li1-C2 120.6(2), N1-Li1-P1 116.1(2), N2-Li1-P1 129.2(2), C2-Li1-P1 88.87(16).

\section{Conclusions}

The $\mathrm{S}_{\mathrm{N}} 2$ reaction of dppm and haloalkanes offer a straightforward access to phosphonium salts, even for secondary or tertiary haloalkanes. The lower yields and long reaction times are partially compensated by the efficient workup of the products and the easy recovery of unreacted dppm. The phosphonium salts can be deprotonated by $\mathrm{KO} t \mathrm{Bu}$ to obtain neutral phosphonium ylides in good yields. 
Further deprotonation with $n$-butyllithium in toluene allows the isolation of lithium complexes containing the corresponding monoanionic ylidic ligands as demonstrated by the synthesis of $\left[\mathrm{Li}\left(\mathrm{Ph}_{2} \mathrm{PCHPPh}_{2} \mathrm{CH}_{2}\right)\right]_{n}$.

The neutral compounds and the monoanionic ylides are promising new ligands for s-block and d-block metal complexes, shielding the metal fragment and offering a sensitive ${ }^{31} \mathrm{P}$ NMR probe.

\section{Experimental Section}

General. All manipulations were carried out in an argon atmosphere under anaerobic conditions. Prior to use, all solvents, except of $\mathrm{CDCl}_{3}$, were thoroughly dried and distilled under an argon atmosphere.

${ }^{1} \mathrm{H},{ }^{31} \mathrm{P}\{\mathrm{H}\},{ }^{13} \mathrm{C}\{\mathrm{H}\}$ and ${ }^{7} \mathrm{Li}\{\mathrm{H}\}$ NMR spectra were recorded at ambient temperature on Bruker AC $200 \mathrm{MHz}$; AC $400 \mathrm{MHz}$ or AC $600 \mathrm{MHz}$ spectrometers. ${ }^{1} \mathrm{H}$ and ${ }^{13} \mathrm{C}\{\mathrm{H}\} \mathrm{NMR}$ spectra were referenced to the residual solvent signals of $98 \%$ perdeuterated $\mathrm{THF}$ or $\mathrm{CDCl}_{3}$ as an internal standard. Melting points were measured with a Reichert-Jung Apparatus Type 302102 and are uncorrected. Elemental analyses were performed on a Leco CHNS-932 at the Institute of Organic Chemistry and Macromolecular Chemistry, FSU Jena.

1,1-Bis(diphenylphosphino)methane (dppm) and the haloalkanes were purchased from Aldrich and used without further purifications. [(Diphenylphosphino)methyl]methyldiphenylphosphonium iodide (1a) was synthesized according to a known procedure. ${ }^{10 \mathrm{~b}}$

Analytical data of [(diphenylphosphino)methyl]methyldiphenylphosphonium iodide (1a).

1a. White solid. ${ }^{1} \mathrm{H} \mathrm{NMR}\left(600 \mathrm{MHz}, \mathrm{CDCl}_{3}\right): \delta_{\mathrm{H}} 2.72\left(3 \mathrm{H}, \mathrm{d},{ }^{2} J_{\mathrm{HP}}=13.5 \mathrm{~Hz}, \mathrm{P}-\mathrm{CH}_{3}\right), 4.15(2 \mathrm{H}$, $\left.\mathrm{d},{ }^{2} J_{\mathrm{HP}}=14.7 \mathrm{~Hz}, \mathrm{P}-\mathrm{CH}_{2}-\mathrm{P}\right), 7.20-7.30(6 \mathrm{H}, \mathrm{m}, \mathrm{CH} \mathrm{Ph}), 7.45-7.57$ (8H, m, CH Ph), 7.59-7.67 $(2 \mathrm{H}, \mathrm{m}, \mathrm{CH} \mathrm{Ph}), 7.78(4 \mathrm{H}, \mathrm{m}, \mathrm{CH} \mathrm{Ph}) .{ }^{13} \mathrm{C}\{\mathrm{H}\} \mathrm{NMR}\left(150.9 \mathrm{MHz}, \mathrm{CDCl}_{3}\right): \delta_{\mathrm{C}} 10.5\left(1 \mathrm{C}, \mathrm{dd},{ }^{1} J_{\mathrm{CP}}\right.$ $\left.=56.7 \mathrm{~Hz},{ }^{3} J_{\mathrm{CP}}=3.1 \mathrm{~Hz}, \mathrm{P}-\mathrm{CH}_{3}\right), 22.5\left(1 \mathrm{C}, \mathrm{dd},{ }^{1} J_{\mathrm{CP}}=51.2 \mathrm{~Hz},{ }^{1} J_{\mathrm{CP}}=34.7 \mathrm{~Hz}, \mathrm{P}-\mathrm{CH}_{2}-\mathrm{P}\right), 119.5$ $\left(2 \mathrm{C}, \mathrm{d},{ }^{1} J_{\mathrm{CP}}=86.5 \mathrm{~Hz}, i-\mathrm{C} \mathrm{Ph}\right), 129.0\left(4 \mathrm{C}, \mathrm{d}, J_{\mathrm{CP}} \sim 7 \mathrm{~Hz}, \mathrm{CH} \mathrm{Ph}\right), 129.9\left(4 \mathrm{C}, \mathrm{d}, J_{\mathrm{CP}}=12.8 \mathrm{~Hz}\right.$, $\mathrm{CH} \mathrm{Ph}$ ), 130.0 (2C, s, p-CH Ph), 132.9 (4C, d, $\left.J_{\mathrm{CP}}=10.3 \mathrm{~Hz}, \mathrm{CH} \mathrm{Ph}\right), 133.6$ (4C, d, $J_{\mathrm{CP}}=21.4$ $\mathrm{Hz}, \mathrm{CH} \mathrm{Ph}), 134.5\left(2 \mathrm{C}\right.$, dd, $\left.{ }^{1} J_{\mathrm{CP}}=10.5 \mathrm{~Hz},{ }^{3} J_{\mathrm{CP}}=8.0 \mathrm{~Hz}, i-\mathrm{C} \mathrm{Ph}\right), 134.7\left(2 \mathrm{C}, \mathrm{d},{ }^{4} J_{\mathrm{CP}}=3.1 \mathrm{~Hz}, p-\right.$ $\mathrm{CH} \mathrm{Ph}) .{ }^{31} \mathrm{P}\{\mathrm{H}\}$ NMR $\left(81 \mathrm{MHz}, \mathrm{CDCl}_{3}\right) \delta_{\mathrm{P}}-22.3\left(1 \mathrm{P}, \mathrm{d},{ }^{2} J_{\mathrm{PP}}=59.7\right), 26.7\left(1 \mathrm{P}, \mathrm{d},{ }^{2} J_{\mathrm{PP}}=59.7\right.$ Hz). Anal. Calcd for $\mathrm{C}_{26} \mathrm{H}_{25} \mathrm{P}_{2} \mathrm{I}$ (526.34): C, 59.33 H, 4.79; I, 24.11\%. Found: C, 59.38; H, 4.77; I, $24.23 \%$. Suitable crystals of the composition $\mathbf{1 a} \cdot 2 \mathrm{CHCl}_{3}$ were obtained from a saturated solution of $1 \mathrm{a}$ in $\mathrm{CHCl}_{3}$ at ambient temperature.

\section{Synthesis of [(diphenylphosphino)methyl] ethyldiphenylphosphonium bromide (1b).}

$\operatorname{Dppm}(5.0 \mathrm{~g}, 13.0 \mathrm{mmol})$ was dissolved in toluene $(60 \mathrm{ml})$ and bromoethane $(8.6 \mathrm{~g}, 78.9 \mathrm{mmol})$ was added. The reaction mixture was heated to $50{ }^{\circ} \mathrm{C}$ for seven days. The resulting white 
precipitate of the composition $\mathbf{1 b} \cdot 0.5$ toluene was collected by filtration, washed with toluene ( 2 $\times 20 \mathrm{ml})$ and dried in vacuum.

1b 0.5 toluene. White solid, yield 51\%, $3.60 \mathrm{~g},{ }^{1} \mathrm{H}$ NMR $\left(200 \mathrm{MHz}, \mathrm{CDCl}_{3}\right): \delta_{\mathrm{H}} 1.08\left(3 \mathrm{H}, \mathrm{dt},{ }^{3} J_{\mathrm{HP}}\right.$ $\left.=20.4 \mathrm{~Hz},{ }^{3} J_{\mathrm{HH}}=7.5 \mathrm{~Hz}, \mathrm{CH}_{3} \mathrm{Et}\right), 2.29\left(1.5 \mathrm{H}, \mathrm{s}, \mathrm{CH}_{3}\right.$ toluene $), 3.35\left(2 \mathrm{H}, \mathrm{dq},{ }^{2} J_{\mathrm{HP}}=12.9 \mathrm{~Hz}\right.$, $\left.{ }^{3} J_{\mathrm{HH}}=7.4 \mathrm{~Hz}, \mathrm{P}-\mathrm{CH}_{2} \mathrm{Et}\right), 4.20\left(2 \mathrm{H}, \mathrm{d},{ }^{2} J_{\mathrm{HP}}=14.3 \mathrm{~Hz}, \mathrm{P}-\mathrm{CH}_{2}-\mathrm{P}\right), 7.02-7.28(8.5 \mathrm{H}, \mathrm{m}, \mathrm{CH} \mathrm{Ph}+$ toluene), 7.38-7.66 (10H, m, $\mathrm{CH} \mathrm{Ph}), 7.70-7.87(4 \mathrm{H}, \mathrm{m}, \mathrm{CH} \mathrm{Ph}) .{ }^{13} \mathrm{C}\{\mathrm{H}\} \mathrm{NMR}(100.6 \mathrm{MHz}$, $\left.\mathrm{CDCl}_{3}\right): \delta_{\mathrm{C}} 6.1\left(1 \mathrm{C}, \mathrm{d},{ }^{2} J_{\mathrm{CP}}=5.0 \mathrm{~Hz}, \mathrm{CH}_{3} \mathrm{Et}\right), 16.9\left(1 \mathrm{C}, \mathrm{dd},{ }^{1} J_{\mathrm{CP}}=51.5 \mathrm{~Hz},{ }^{3} J_{\mathrm{CP}}=3.8 \mathrm{~Hz}, \mathrm{CH}_{2}\right.$ Et), $20.2\left(1 \mathrm{C}, \mathrm{dd},{ }^{1} J_{\mathrm{CP}}=49.8 \mathrm{~Hz},{ }^{1} J_{\mathrm{CP}}=34.1 \mathrm{~Hz}, \mathrm{P}-\mathrm{CH}_{2}-\mathrm{P}\right), 21.3\left(0.5 \mathrm{C}, \mathrm{s}, \mathrm{CH}_{3}\right.$ toluene), 117.4 $\left(2 \mathrm{C}, \mathrm{d},{ }^{1} J_{\mathrm{CP}}=84.0 \mathrm{~Hz}, i-\mathrm{C} \mathrm{Ph}\right), 125.1(0.5 \mathrm{C}, \mathrm{s}, p-\mathrm{CH}$ toluene), 128.0 (1C, s, $m$-CH toluene), $128.7\left(4 \mathrm{C}, \mathrm{d}, J_{\mathrm{CP}}=8.0 \mathrm{~Hz}, \mathrm{CH} \mathrm{Ph}\right), 128.8$ (1C, s, o-CH toluene), 129.6 (2C, s, p-CH Ph), 129.7 $\left(4 \mathrm{C}, \mathrm{d}, J_{\mathrm{CP}}=12.4 \mathrm{~Hz}, \mathrm{CH} \mathrm{Ph}\right), 133.2\left(4 \mathrm{C}, \mathrm{d},{ }^{2} J_{\mathrm{CP}}=22.1 \mathrm{~Hz}, \mathrm{CH} \mathrm{Ph}\right), 133.3\left(4 \mathrm{C}, \mathrm{dd},{ }^{2} J_{\mathrm{CP}} \sim 10\right.$ $\left.\mathrm{Hz},{ }^{4} J_{\mathrm{CP}}=1.2 \mathrm{~Hz}, o-\mathrm{CH} \mathrm{Ph}\right), 134.4\left(2 \mathrm{C}, \mathrm{d},{ }^{4} J_{\mathrm{CP}}=2.6 \mathrm{~Hz}, p-\mathrm{CH} \mathrm{Ph}\right), 134.8\left(2 \mathrm{C}, \mathrm{dd},{ }^{1} J_{\mathrm{CP}}=11.4\right.$ $\left.\mathrm{Hz},{ }^{3} J_{\mathrm{CP}}=7.8 \mathrm{~Hz}, i-\mathrm{C} \mathrm{Ph}\right) 137.6\left(0.5 \mathrm{C}, \mathrm{s}, i\right.$-C toluene). ${ }^{31} \mathrm{P}\{\mathrm{H}\} \mathrm{NMR}\left(81 \mathrm{MHz}, \mathrm{CDCl}_{3}\right) \delta_{\mathrm{P}}-23.2$ $\left(1 \mathrm{P}, \mathrm{d},{ }^{2} J_{\mathrm{PP}}=58.2 \mathrm{~Hz}\right), 33.8\left(1 \mathrm{P}, \mathrm{d},{ }^{2} J_{\mathrm{PP}}=58.2 \mathrm{~Hz}\right)$. For X-ray diffraction experiments and elemental analysis, crystals of the composition $\mathbf{1 b} \cdot$ toluene, obtained directly from the reaction mixture at ambient temperature, were used. Anal. Calcd for $\mathrm{C}_{34} \mathrm{H}_{35} \mathrm{P}_{2} \mathrm{Br}$ (585.51): $\mathrm{C}, 69.75 ; \mathrm{H}$, 6.03; Br, 13.65\%. Found: C, 69.69; H, 5.84; Br 13.88\%. This crop still contained a very small amount of crystals of the composition $\mathbf{1 b} \cdot 0.5$ toluene, suitable for X-ray diffraction measurements.

\section{Synthesis of [(diphenylphosphino)methyl]isopropyldiphenylphosphonium iodide (1c).}

Dppm (4.4 g, $11.4 \mathrm{mmol})$ was dissolved in toluene $(50 \mathrm{ml})$ and 2-iodopropane $(8.0 \mathrm{~g}$, $47.1 \mathrm{mmol}$ ) was added. The reaction mixture was heated to $80{ }^{\circ} \mathrm{C}$ for seven days. The resulting white precipitate of the composition $1 \mathbf{c} \cdot$ toluene was collected by filtration, washed with toluene $(2 \times 20 \mathrm{ml})$ and dried in vacuum.

1c·toluene. White solid, yield 37\%, $2.6 \mathrm{~g},{ }^{1} \mathrm{H}$ NMR $\left(200 \mathrm{MHz}, \mathrm{CDCl}_{3}\right): \delta_{\mathrm{H}} 1.18\left(6 \mathrm{H}, \mathrm{dd},{ }^{3} J_{\mathrm{HP}}=\right.$ $\left.18.9 \mathrm{~Hz},{ }^{3} J_{\mathrm{HH}}=7.0 \mathrm{~Hz}, \mathrm{CH}_{3} i \mathrm{Pr}\right), 2.31\left(3 \mathrm{H}, \mathrm{s}, \mathrm{CH}_{3}\right.$ toluene $), 4.05\left(2 \mathrm{H}, \mathrm{d},{ }^{2} J_{\mathrm{HP}}=13.0 \mathrm{~Hz}, \mathrm{P}-\mathrm{CH}_{2^{-}}\right.$ P), $4.20\left(1 \mathrm{H}, \mathrm{dsept},{ }^{2} J_{\mathrm{HP}}=11.6 \mathrm{~Hz},{ }^{3} J_{\mathrm{HH}}=7.1 \mathrm{~Hz}, \mathrm{P}-\mathrm{CH}_{2} \mathrm{Et}\right), 7.00-7.30(11 \mathrm{H}, \mathrm{m}, \mathrm{CH} \mathrm{Ph}+$ toluene), 7.40-7.90 $(14 \mathrm{H}, \mathrm{m}, \mathrm{CH}) .{ }^{13} \mathrm{C}\{\mathrm{H}\} \mathrm{NMR}\left(50.3 \mathrm{MHz}, \mathrm{CDCl}_{3}\right): \delta_{\mathrm{C}} 15.6\left(2 \mathrm{C}, \mathrm{d},{ }^{2} J_{\mathrm{CP}}=1.4\right.$ $\left.\mathrm{Hz}, \mathrm{CH}_{3} i \mathrm{Pr}\right), 18.7\left(1 \mathrm{C}, \mathrm{dd},{ }^{1} J_{\mathrm{CP}}=48.7 \mathrm{~Hz},{ }^{1} J_{\mathrm{CP}}=33.3 \mathrm{~Hz}, \mathrm{P}-\mathrm{CH}_{2}-\mathrm{P}\right), 21.4\left(1 \mathrm{C}, \mathrm{s}, \mathrm{CH}_{3}\right.$ toluene), $23.9\left(1 \mathrm{C}, \mathrm{dd},{ }^{1} J_{\mathrm{CP}}=46.7 \mathrm{~Hz},{ }^{3} J_{\mathrm{CP}}=2.2 \mathrm{~Hz}, \mathrm{CH} i \mathrm{Pr}\right), 115.0\left(2 \mathrm{C}, \mathrm{d},{ }^{1} J_{\mathrm{CP}}=81.9 \mathrm{~Hz}, i-\mathrm{C} \mathrm{Ph}\right), 125.2$ (1C, s, $p-\mathrm{CH}$ toluene), 128.2 (2C, s, $m-\mathrm{CH}$ toluene), $128.8\left(4 \mathrm{C}, \mathrm{d}, J_{\mathrm{CP}}=8.9 \mathrm{~Hz}, \mathrm{CH} \mathrm{Ph}\right), 128.9$ (2C, s, o-CH toluene), $129.7(2 \mathrm{C}, \mathrm{s}, p-\mathrm{CH} \mathrm{Ph}), 129.8$ (4C, d, $\left.J_{\mathrm{CP}}=11.8 \mathrm{~Hz}, \mathrm{CH} \mathrm{Ph}\right), 133.3(4 \mathrm{C}$, $\left.\mathrm{d}, J_{\mathrm{CP}}=22.4 \mathrm{~Hz}, \mathrm{CH} \mathrm{Ph}\right), 134.1\left(4 \mathrm{C}, \mathrm{dd},{ }^{2} J_{\mathrm{CP}}=8.5 \mathrm{~Hz},{ }^{4} J_{\mathrm{CP}}=1.4 \mathrm{~Hz}, o-\mathrm{CH} \mathrm{Ph}\right), 134.6(2 \mathrm{C}, \mathrm{dd}$, $\left.{ }^{1} J_{\mathrm{CP}}=10.7 \mathrm{~Hz},{ }^{3} J_{\mathrm{CP}}=7.5 \mathrm{~Hz}, i-\mathrm{C} \mathrm{Ph}\right), 134.7\left(2 \mathrm{C}, \mathrm{d},{ }^{4} J_{\mathrm{CP}}=3.0 \mathrm{~Hz}, p-\mathrm{CH} \mathrm{Ph}\right), 137.8(1 \mathrm{C}, \mathrm{s}, i-\mathrm{C}$ toluene). ${ }^{31} \mathrm{P}\{\mathrm{H}\} \mathrm{NMR}\left(81 \mathrm{MHz}, \mathrm{CDCl}_{3}\right) \delta_{\mathrm{P}}-24.6\left(1 \mathrm{P}, \mathrm{d},{ }^{2} J_{\mathrm{PP}}=58.7 \mathrm{~Hz}\right), 38.1\left(1 \mathrm{P}, \mathrm{d},{ }^{2} J_{\mathrm{PP}}=58.7\right.$ Hz). Anal. Calcd for $\mathrm{C}_{35} \mathrm{H}_{37} \mathrm{P}_{2} \mathrm{I}$ (646.53): C, 65.02; H, 5.77; I, 19.63\%. Found: C, 65.06; H, 5.72, $19.54 \%$. Suitable crystals of the composition $\mathbf{1 c} \cdot \mathrm{CHCl}_{3}$ for X-ray diffraction experiments were obtained by slow diffusion of $\mathrm{Et}_{2} \mathrm{O}$ into a saturated solution of $1 \mathrm{c} \cdot$ toluene in $\mathrm{CHCl}_{3}$. 
Synthesis of [(diphenylphosphino)methyl]diphenyl[(2,4,6-trimethylphenyl)methyl]phosphonium bromide (1d). Dppm (3.59 g, $9.34 \mathrm{mmol}$ ) was dissolved in toluene (40 ml) and 2(bromomethyl)-1,3,5-trimethylbenzene $(1.99 \mathrm{~g}, 9.34 \mathrm{mmol})$ was added. The reaction mixture was heated to $80^{\circ} \mathrm{C}$ for two days. The resulting white precipitate of $\mathbf{1 d}$ was collected by filtration, washed with toluene $(2 \times 20 \mathrm{ml})$ and dried in vacuum.

1d. White solid, yield 99\%, $5.53 \mathrm{~g}, \mathrm{mp} 240-241{ }^{\circ} \mathrm{C} ;{ }^{1} \mathrm{H}$ NMR $\left(600 \mathrm{MHz}, \mathrm{CDCl}_{3}\right): \delta_{\mathrm{H}} 1.68(6 \mathrm{H}, \mathrm{s}$, $\left.o-\mathrm{CH}_{3} \mathrm{Mes}\right), 2.10\left(3 \mathrm{H}, \mathrm{d},{ }^{7} J_{\mathrm{HP}}=2.7 \mathrm{~Hz}, p-\mathrm{CH}_{3} \mathrm{Mes}\right), 4.45\left(2 \mathrm{H}, \mathrm{d},{ }^{2} J_{\mathrm{HP}}=13.8 \mathrm{~Hz}, \mathrm{P}-\mathrm{CH}_{2}-\mathrm{P}\right)$, $4.79\left(2 \mathrm{H}, \mathrm{d},{ }^{2} J_{\mathrm{HP}}=14.5 \mathrm{~Hz}, \mathrm{P}-\mathrm{CH}_{2}\right), 6.56(2 \mathrm{H}, \mathrm{s}, m-\mathrm{CH} \mathrm{Mes}), 7.18-7.23(6 \mathrm{H}, \mathrm{m}, \mathrm{CH} \mathrm{Ph}), 7.26-$ $7.32(4 \mathrm{H}, \mathrm{m}, \mathrm{CH} \mathrm{Ph}), 7.50-7.58(10 \mathrm{H}, \mathrm{m}, \mathrm{CH} \mathrm{Ph}) .{ }^{13} \mathrm{C}\{\mathrm{H}\} \mathrm{NMR}\left(100.6 \mathrm{MHz}, \mathrm{CDCl}_{3}\right): \delta_{\mathrm{C}} 20.7$ $\left(1 \mathrm{C}, \mathrm{d},{ }^{6} J_{\mathrm{CP}}=1.4 \mathrm{~Hz}, p-\mathrm{CH}_{3} \mathrm{Mes}\right), 21.0\left(2 \mathrm{C}, \mathrm{d},{ }^{4} J_{\mathrm{CP}}=1.0 \mathrm{~Hz}, o-\mathrm{CH}_{3} \mathrm{Mes}\right), 22.0\left(1 \mathrm{C}, \mathrm{dd},{ }^{1} J_{\mathrm{CP}}=\right.$ $\left.47.7 \mathrm{~Hz},{ }^{1} J_{\mathrm{CP}}=32.9 \mathrm{~Hz}, \mathrm{P}-\mathrm{CH}_{2}-\mathrm{P}\right), 30.3\left(1 \mathrm{C}, \mathrm{d},{ }^{1} J_{\mathrm{CP}}=45.4 \mathrm{~Hz}, \mathrm{P}-\mathrm{CH}_{2}\right), 116.9\left(2 \mathrm{C}, \mathrm{d},{ }^{1} J_{\mathrm{CP}}=82.6\right.$ $\mathrm{Hz}, i-\mathrm{C} \mathrm{Ph}), 123.1\left(1 \mathrm{C}, \mathrm{d}, J_{\mathrm{CP}}=9.5 \mathrm{~Hz}, \mathrm{C} \mathrm{Mes}\right), 128.9\left(4 \mathrm{C}, \mathrm{d}, J_{\mathrm{CP}}=8.6 \mathrm{~Hz}, \mathrm{CH} \mathrm{Ph}\right), 129.2(4 \mathrm{C}$, $\left.\mathrm{d}, J_{\mathrm{CP}}=12.1 \mathrm{~Hz}, \mathrm{CH} \mathrm{Ph}\right), 129.5\left(2 \mathrm{C}, \mathrm{d},{ }^{4} J_{\mathrm{CP}}=3.6 \mathrm{~Hz}, m-\mathrm{CH} \mathrm{Mes}\right), 129.7$ (2C, s, $\left.p-\mathrm{CH} \mathrm{Ph}\right)$, $133.4\left(4 \mathrm{C}, \mathrm{d}, J_{\mathrm{CP}}=22.6 \mathrm{~Hz}, \mathrm{CH} \mathrm{Ph}\right), 134.0\left(4 \mathrm{C}, \mathrm{dd},{ }^{2} J_{\mathrm{CP}}=8.9 \mathrm{~Hz},{ }^{4} J_{\mathrm{CP}}=2.7 \mathrm{~Hz}, o-\mathrm{CH} \mathrm{Ph}\right)$, $134.4\left(2 \mathrm{C}, \mathrm{d},{ }^{4} J_{\mathrm{CP}}=2.9 \mathrm{~Hz}, p-\mathrm{CH} \mathrm{Ph}\right), 135.0\left(2 \mathrm{C}, \mathrm{dd},{ }^{1} J_{\mathrm{CP}}=11.2 \mathrm{~Hz},{ }^{3} J_{\mathrm{CP}}=8.0 \mathrm{~Hz}, i-\mathrm{C} \mathrm{Ph}\right)$, $137.7\left(1 \mathrm{C}, \mathrm{d}, J_{\mathrm{CP}}=4.4 \mathrm{~Hz}, \mathrm{C} \mathrm{Mes}\right), 137.8\left(2 \mathrm{C}, \mathrm{d},{ }^{3} J_{\mathrm{CP}}=5.4 \mathrm{~Hz}, o-\mathrm{C} \mathrm{Mes}\right) .{ }^{31} \mathrm{P}\{\mathrm{H}\} \mathrm{NMR}$ $\left(161.9 \mathrm{MHz}, \mathrm{CDCl}_{3}\right) \delta_{\mathrm{P}}-23.8\left(1 \mathrm{P}, \mathrm{d},{ }^{2} J_{\mathrm{PP}}=61.3 \mathrm{~Hz}\right), 25.8\left(1 \mathrm{P}, \mathrm{d},{ }^{2} J_{\mathrm{PP}}=61.3 \mathrm{~Hz}\right)$. Anal. Calcd for $\mathrm{C}_{35} \mathrm{H}_{35} \mathrm{P}{ }_{2} \mathrm{Br}$ (597.52): C, 70.36 H, 5.90; Br, 13.37\%. Found: C, 70.20; H, 5.96; Br, 13.51\%. Suitable crystals of $\mathbf{1 d}$ for X-ray diffraction experiments were obtained by slow diffusion of $\mathrm{Et}_{2} \mathrm{O}$ into a saturated solution of $\mathbf{1 d}$ in $\mathrm{CHCl}_{3}$.

\section{Synthesis of tert-butyl[(diphenylphosphino)methyl]diphenylphosphonium bromide (1e) and} formation of methyldiphenylphosphonium bromide (3). Dppm (6.5 g, $16.9 \mathrm{mmol})$ was dissolved in toluene $(50 \mathrm{ml})$ and 2-bromo-2-methylpropane $(10.0 \mathrm{~g}, 73.0 \mathrm{mmol})$ was added. The reaction mixture was heated to $70{ }^{\circ} \mathrm{C}$ for three weeks. The resulting white precipitate of $1 \mathrm{e}$ was collected by filtration, washed with toluene $(2 \times 20 \mathrm{ml})$ and dried in vacuum.

1e. White solid, yield $19 \%, 1.64 \mathrm{~g}$. Suitable crystals of $\mathbf{1 e} \cdot 2 \mathrm{CH}_{2} \mathrm{Cl}_{2}$ for $\mathrm{X}$-ray diffraction experiments were obtained by cooling a saturated solution of $1 \mathbf{e}$ in a mixture of $\mathrm{Et}_{2} \mathrm{O}$ and dichloromethane from ambient temperature to $-20{ }^{\circ} \mathrm{C} .{ }^{1} \mathrm{H}$ NMR $\left(200 \mathrm{MHz}, \mathrm{CDCl}_{3}\right): \delta_{\mathrm{H}} 1.47(9 \mathrm{H}$, $\left.\mathrm{d},{ }^{3} J_{\mathrm{HP}}=17.1 \mathrm{~Hz}, \mathrm{CH}_{3} t \mathrm{Bu}\right), 3.95\left(2 \mathrm{H}, \mathrm{d},{ }^{2} J_{\mathrm{HP}}=12.3 \mathrm{~Hz}, \mathrm{P}-\mathrm{CH}_{2}-\mathrm{P}\right), 7.1-7.8(20 \mathrm{H}, \mathrm{m}, \mathrm{CH} \mathrm{Ph})$. ${ }^{13} \mathrm{C}\{\mathrm{H}\}$ NMR $\left(50.3 \mathrm{MHz}, \mathrm{CDCl}_{3}\right): \delta_{\mathrm{C}} 18.4\left(1 \mathrm{C}, \mathrm{dd},{ }^{1} J_{\mathrm{CP}}=46.2 \mathrm{~Hz},{ }^{3} J_{\mathrm{CP}}=35.7 \mathrm{~Hz}, \mathrm{P}-\mathrm{CH}_{2}-\mathrm{P}\right)$, $26.5\left(3 \mathrm{C}, \mathrm{s}, \mathrm{CH}_{3} t \mathrm{Bu}\right), 34.4\left(1 \mathrm{C}, \mathrm{d},{ }^{1} J_{\mathrm{CP}}=42.0 \mathrm{~Hz}, \mathrm{P}-\mathrm{C} t \mathrm{Bu}\right), 116.4\left(2 \mathrm{C}, \mathrm{dd},{ }^{1} J_{\mathrm{CP}}=79.5 \mathrm{~Hz},{ }^{3} J_{\mathrm{CP}}\right.$ $=1.8 \mathrm{~Hz}, i-\mathrm{C} \mathrm{Ph}), 128.8\left(4 \mathrm{C}, \mathrm{d}, J_{\mathrm{CP}}=8.4 \mathrm{~Hz}, \mathrm{CH} \mathrm{Ph}\right), 129.6\left(4 \mathrm{C}, \mathrm{d}, J_{\mathrm{CP}}=11.7 \mathrm{~Hz}, \mathrm{CH} \mathrm{Ph}\right)$, $129.6(2 \mathrm{C}, \mathrm{s}, p-\mathrm{CH} \mathrm{Ph}), 133.2\left(4 \mathrm{C}, \mathrm{d}, J_{\mathrm{CP}}=22.7 \mathrm{~Hz}, \mathrm{CH} \mathrm{Ph}\right), 134.2\left(4 \mathrm{C}, \mathrm{dd},{ }^{2} J_{\mathrm{CP}}=8.2 \mathrm{~Hz},{ }^{4} J_{\mathrm{CP}}=\right.$ $1.8 \mathrm{~Hz}, o-\mathrm{CH} \mathrm{Ph}), 134.5\left(2 \mathrm{C}, \mathrm{d},{ }^{4} J_{\mathrm{CP}}=2.7 \mathrm{~Hz}, p-\mathrm{CH} \mathrm{Ph}\right), 134.8\left(2 \mathrm{C}, \mathrm{dd},{ }^{1} J_{\mathrm{CP}}=12.1 \mathrm{~Hz},{ }^{3} J_{\mathrm{CP}}=\right.$ $7.6 \mathrm{~Hz}, i-\mathrm{C} \mathrm{Ph}) .{ }^{31} \mathrm{P}\{\mathrm{H}\} \mathrm{NMR}\left(81 \mathrm{MHz}, \mathrm{CDCl}_{3}\right) \delta_{\mathrm{P}}-23.0\left(1 \mathrm{P}, \mathrm{d},{ }^{2} J_{\mathrm{PP}}=60.5 \mathrm{~Hz}\right), 41.2\left(1 \mathrm{P}, \mathrm{d},{ }^{2} J_{\mathrm{PP}}\right.$ $=60.5 \mathrm{~Hz})$. MS $\left\{\mathrm{ESI}\right.$ in $\left.\mathrm{CHCl}_{3} / \mathrm{CH}_{3} \mathrm{OH}\right\}(\mathrm{m} / \mathrm{z}, \%): 441.2\left(\mathrm{M}^{+}, 100\right)$. HRMS: calcd for the cation $\mathrm{C}_{29} \mathrm{H}_{31} \mathrm{P}_{2}, 441.1901$; found 441.1887 .

Further crops of product 1e were obtained by heating of the mother liquor of the reaction for additional weeks, but these fractions contained by-products. A few crystals of one of those by- 
products grew at the wall of the Schlenk tube right above the solvent level, when the reaction mixture was kept undisturbed at ambient temperature for several days after weeks of heating. The compound was identified as $\left[\mathrm{Ph}_{2} \mathrm{MePH}\right]^{+} \mathrm{Br}^{-}(3)$ by X-ray diffraction experiments.

3. Colorless crystals. ${ }^{1} \mathrm{H}$ NMR $\left(400 \mathrm{MHz}, \mathrm{CDCl}_{3}\right): \delta_{\mathrm{H}} 2.57\left(3 \mathrm{H}, \mathrm{d},{ }^{2} J_{\mathrm{HP}}=14.6 \mathrm{~Hz}, \mathrm{CH}_{3}\right), 7.56$ $(4 \mathrm{H}, \mathrm{m}, \mathrm{CH} \mathrm{Ph}), 7.67(2 \mathrm{H}, \mathrm{m}, \mathrm{CH} \mathrm{Ph}), 7.97(4 \mathrm{H}, \mathrm{m}, \mathrm{CH} \mathrm{Ph})$, the signal of the P-H group was not observed, probably due to exchange with $\mathrm{D}_{2} \mathrm{O}$ or $\mathrm{DCl}$ present as impurities in the used $\mathrm{CDCl}_{3}$. ${ }^{13} \mathrm{C}\{\mathrm{H}\}$ NMR $\left(100.6 \mathrm{MHz}, \mathrm{CDCl}_{3}\right): \delta_{\mathrm{C}} 7.3\left(1 \mathrm{C}, \mathrm{d},{ }^{1} J_{\mathrm{CP}}=53.3 \mathrm{~Hz}, \mathrm{P}-\mathrm{CH}_{3}\right), 117.6\left(2 \mathrm{C}, \mathrm{d},{ }^{1} J_{\mathrm{CP}}=\right.$ $83.0 \mathrm{~Hz}, i$-C Ph), 130.1 (4C, d, $\left.J_{\mathrm{CP}}=13.0 \mathrm{~Hz}, \mathrm{CH} \mathrm{Ph}\right), 133.1\left(4 \mathrm{C}, \mathrm{d}, J_{\mathrm{CP}}=10.9 \mathrm{~Hz}, \mathrm{CH} \mathrm{Ph}\right)$, $134.7\left(2 \mathrm{C}, \mathrm{d}, J_{\mathrm{CP}}=2.4 \mathrm{~Hz}, p-\mathrm{CH} \mathrm{Ph}\right) .{ }^{31} \mathrm{P}\{\mathrm{H}\} \mathrm{NMR}\left(81 \mathrm{MHz}, \mathrm{CDCl}_{3}\right) \delta_{\mathrm{P}}-2.4(\mathrm{~s})$.

Synthesis of [(diphenylphosphino)methylene]methyldiphenylphosphorane (2a). A solution of $\mathrm{KO} t \mathrm{Bu}$ in THF $(6.8 \mathrm{ml}, 1 \mathrm{M})$ was added to a stirred suspension of $\mathbf{1 a}(3.6 \mathrm{~g}, 6.84 \mathrm{mmol})$ in THF $(40 \mathrm{ml})$ at ambient temperature. The resulting mixture was stirred for an additional hour and filtered over diatomaceous earth to remove precipitated $\mathrm{KBr}$. The obtained yellow solution was reduced to dryness and dried in vacuum. The resulting residue was taken up in diethyl ether (40 $\mathrm{ml}$ ) and filtered again to remove a small amount of solid. Afterwards, the yellow solution was reduced to dryness. The remaining oil started to crystallize after addition of heptane $(30 \mathrm{ml})$. The mixture was vigorously stirred until all oil has transformed in a pale yellow solid, which was isolated by filtration and dried in vacuum. 2a. Pale yellow solid, yield 78\%, $2.12 \mathrm{~g}$. ${ }^{1} \mathrm{H}$ NMR $\left(600 \mathrm{MHz},\left[\mathrm{D}_{8}\right] \mathrm{THF}\right): \delta_{\mathrm{H}} 1.23\left(1 \mathrm{H}, \mathrm{dd},,^{2} J_{\mathrm{HP}}=11.4 \mathrm{~Hz},{ }^{2} J_{\mathrm{HP}}=1.6 \mathrm{~Hz}, \mathrm{P}-\mathrm{CH}=\mathrm{P}\right), 2.17(3 \mathrm{H}, \mathrm{d}$, $\left.{ }^{2} J_{\mathrm{HP}}=12.7 \mathrm{~Hz}, \mathrm{P}-\mathrm{CH}_{3}\right), 7.10(2 \mathrm{H}, \mathrm{m}, \mathrm{CH} \mathrm{Ph}), 7.18$ (4H, m, CH Ph ), 7.39 (4H, m, CH Ph ), 7.45 $(2 \mathrm{H}, \mathrm{m}, \mathrm{CH} \mathrm{Ph}), 7.52(4 \mathrm{H}, \mathrm{m}, \mathrm{CH} \mathrm{Ph}), 7.71(4 \mathrm{H}, \mathrm{m}, \mathrm{CH} \mathrm{Ph}) .{ }^{13} \mathrm{C}\{\mathrm{H}\} \mathrm{NMR}(150.9 \mathrm{MHz}$, $\left.\left[\mathrm{D}_{8}\right] \mathrm{THF}\right): \delta_{\mathrm{C}} 8.5\left(1 \mathrm{C}, \mathrm{dd},{ }^{1} J_{\mathrm{CP}}=118.9 \mathrm{~Hz},{ }^{1} J_{\mathrm{CP}}=11.2 \mathrm{~Hz}, \mathrm{P}-\mathrm{CH}=\mathrm{P}\right), 14.2\left(1 \mathrm{C}, \mathrm{dd},{ }^{1} J_{\mathrm{CP}}=67.2\right.$ $\left.\mathrm{Hz},{ }^{3} J_{\mathrm{CP}}=12.9 \mathrm{~Hz}, \mathrm{CH}_{3}\right), 126.7(2 \mathrm{C}, \mathrm{s}, p-\mathrm{CH} \mathrm{Ph}), 128.0\left(4 \mathrm{C}, \mathrm{d}, J_{\mathrm{CP}}=6.0 \mathrm{~Hz}, \mathrm{CH} \mathrm{Ph}\right), 129.0$ $\left(4 \mathrm{C}, \mathrm{d}, J_{\mathrm{CP}}=11.5 \mathrm{~Hz}, \mathrm{CH} \mathrm{Ph}\right), 131.5\left(2 \mathrm{C}, \mathrm{d},{ }^{4} J_{\mathrm{CP}}=2.3 \mathrm{~Hz}, p-\mathrm{CH} \mathrm{Ph}\right), 132.3\left(4 \mathrm{C}, \mathrm{d}, J_{\mathrm{CP}}=18.6\right.$ $\mathrm{Hz}, \mathrm{CH} \mathrm{Ph}), 132.3\left(4 \mathrm{C}, \mathrm{d}, J_{\mathrm{CP}}=9.3 \mathrm{~Hz}, \mathrm{CH} \mathrm{Ph}\right), 135.4\left(2 \mathrm{C}, \mathrm{dd},{ }^{1} J_{\mathrm{CP}}=83.4 \mathrm{~Hz},{ }^{3} J_{\mathrm{CP}}=2.85 \mathrm{~Hz}, i-\right.$ $\mathrm{C} \mathrm{Ph}), 148.5$ (2C, pseudo-t, $\left.{ }^{1} J_{\mathrm{CP}}={ }^{3} J_{\mathrm{CP}}=10.5 \mathrm{~Hz}, i-\mathrm{C} \mathrm{Ph}\right) .{ }^{31} \mathrm{P}\{\mathrm{H}\} \mathrm{NMR}\left(81 \mathrm{MHz},\left[\mathrm{D}_{8}\right] \mathrm{THF}\right) \delta_{\mathrm{P}}-$ $16.3\left(1 \mathrm{P}, \mathrm{d},{ }^{2} J_{\mathrm{PP}}=158.3 \mathrm{~Hz}\right), 19.4\left(1 \mathrm{P}, \mathrm{d},{ }^{2} J_{\mathrm{PP}}=158.3 \mathrm{~Hz}\right)$. Anal. Calcd for $\mathrm{C}_{26} \mathrm{H}_{24} \mathrm{P}_{2}(398.426)$ : C, 78.38; H, 6.07\%. Found: C, 78.36; H, 6.04\%. Suitable crystals of 2a for X-ray diffraction experiments were obtained by cooling a saturated solution in $\mathrm{Et}_{2} \mathrm{O}$ from ambient temperature to $20{ }^{\circ} \mathrm{C}$.

Synthesis of [(diphenylphosphino)methylene]ethyldiphenylphosphorane (2b). A solution of $\mathrm{KO} t \mathrm{Bu}$ in $\mathrm{THF}(5.7 \mathrm{ml}, 1 \mathrm{M})$ was added to a stirred suspension of $\mathbf{1 b} \cdot 0.5$ toluene $(3.07 \mathrm{~g}$, $5.69 \mathrm{mmol})$ in THF $(35 \mathrm{ml})$ at ambient temperature. The reaction mixture was stirred for an additional hour and filtered over diatomaceous earth to remove precipitated $\mathrm{KBr}$. The obtained yellow solution was reduced to dryness and dried in vacuum. The resulting solid foam was taken up in diethyl ether $(20 \mathrm{ml})$ and the suspension obtained was stirred for 30 minutes. The formed product was afterwards isolated by filtration and dried in vacuum. A second crop of product was 
obtained in form of well shaped pale yellow crystals, suitable for X-ray diffraction experiments, by storing the mother liquor at $-10{ }^{\circ} \mathrm{C}$ over night.

2b. Pale yellow crystals, yield 81\%, $1.89 \mathrm{~g}, \mathrm{mp} 120-123{ }^{\circ} \mathrm{C} .{ }^{1} \mathrm{H}$ NMR $\left(200 \mathrm{MHz},\left[\mathrm{D}_{8}\right] \mathrm{THF}\right): \delta_{\mathrm{H}}$ 1.00-1.25 (4H, m, P-CH=P $\left.+\mathrm{CH}_{3}\right), 2.57\left(2 \mathrm{H}, \mathrm{dq},{ }^{2} J_{\mathrm{HP}}=12.5 \mathrm{~Hz},{ }^{3} J_{\mathrm{HH}}=7.3 \mathrm{~Hz}, \mathrm{P}-\mathrm{CH}_{2}\right), 7.00-$ 7.25 (6H, m, CH Ph ), 7.30-7.48 (6H, m, CH Ph), 7.48-7.62 (4H, m, CH Ph), 7.68-7.84 (4H, m, $\mathrm{CH} \mathrm{Ph}) .{ }^{13} \mathrm{C}\{\mathrm{H}\}$ NMR $\left(150.9 \mathrm{MHz},\left[\mathrm{D}_{8}\right] \mathrm{THF}\right): \delta_{\mathrm{C}} 3.9\left(1 \mathrm{C}, \mathrm{dd},{ }^{1} J_{\mathrm{CP}}=117.3 \mathrm{~Hz},{ }^{1} J_{\mathrm{CP}}=10.6 \mathrm{~Hz}\right.$, $\mathrm{P}-\mathrm{CH}=\mathrm{P}), 7.1\left(1 \mathrm{C}\right.$, pseudo-t, $\left.{ }^{2} J_{\mathrm{CP}}={ }^{4} J_{\mathrm{CP}}=3.7 \mathrm{~Hz}, \mathrm{CH}_{3} \mathrm{Et}\right), 20.6\left(1 \mathrm{C}, \mathrm{dd},{ }^{1} J_{\mathrm{CP}}=63.8 \mathrm{~Hz},{ }^{3} J_{\mathrm{CP}}=\right.$ $\left.8.5 \mathrm{~Hz}, \mathrm{CH}_{2} \mathrm{Et}\right), 126.7$ (2C, s, $\left.p-\mathrm{CH} \mathrm{Ph}\right), 128.0$ (4C, d, $\left.J_{\mathrm{CP}}=6.2 \mathrm{~Hz}, \mathrm{CH} \mathrm{Ph}\right), 129.0\left(4 \mathrm{C}, \mathrm{d}, J_{\mathrm{CP}}=\right.$ $10.9 \mathrm{~Hz}, \mathrm{CH} \mathrm{Ph}), 131.6\left(2 \mathrm{C}, \mathrm{d},{ }^{4} J_{\mathrm{CP}}=2.0 \mathrm{~Hz}, p-\mathrm{CH} \mathrm{Ph}\right), 132.3\left(4 \mathrm{C}, \mathrm{d}, J_{\mathrm{CP}}=18.7 \mathrm{~Hz}, \mathrm{CH} \mathrm{Ph}\right)$, $132.7\left(4 \mathrm{C}, \mathrm{d}, J_{\mathrm{CP}}=8.9 \mathrm{~Hz}, \mathrm{CH} \mathrm{Ph}\right), 133.9\left(2 \mathrm{C}, \mathrm{dd},{ }^{1} J_{\mathrm{CP}}=81.4 \mathrm{~Hz},{ }^{3} J_{\mathrm{CP}}=3.5 \mathrm{~Hz}, i-\mathrm{C} \mathrm{Ph}\right), 148.6$ (2C, pseudo-t, $\left.{ }^{1} J_{\mathrm{CP}}={ }^{3} J_{\mathrm{CP}}=10.6 \mathrm{~Hz}, i-\mathrm{C} \mathrm{Ph}\right) .{ }^{31} \mathrm{P}\{\mathrm{H}\} \mathrm{NMR}\left(81 \mathrm{MHz},\left[\mathrm{D}_{8}\right] \mathrm{THF}\right) \delta_{\mathrm{P}}-16.3(1 \mathrm{P}, \mathrm{d}$, $\left.{ }^{2} J_{\mathrm{PP}}=150.7 \mathrm{~Hz}\right), 27.7\left(1 \mathrm{P}, \mathrm{d},{ }^{2} J_{\mathrm{PP}}=150.7 \mathrm{~Hz}\right)$. Anal. Calcd for $\mathrm{C}_{27} \mathrm{H}_{26} \mathrm{P}_{2}$ (412.45): C, 78.63; $\mathrm{H}$, $6.35 \%$. Found: C, 78.59; H, 6.33\%.

Synthesis of [(diphenylphosphino)methylene]isopropyldiphenylphosphorane (2c). A solution of $\mathrm{KO} t \mathrm{Bu}$ in $\mathrm{THF}(1.8 \mathrm{ml}, 1 \mathrm{M})$ was added to a stirred suspension of $1 \mathrm{c} \cdot$ toluene $(1.1 \mathrm{~g}$, $1.70 \mathrm{mmol})$ in THF $(15 \mathrm{ml})$ at ambient temperature. The resulting mixture was stirred for an additional hour and filtered over diatomaceous earth to remove precipitated KI. The obtained yellow solution was reduced to dryness and dried in vacuum. The resulting solid foam was taken up in diethyl ether $(20 \mathrm{ml})$ and the solution was filtered and stored at $-20{ }^{\circ} \mathrm{C}$ over night. (The solution tends to oversaturate and in some cases lower temperatures and longer storage times were necessary to induce crystallization.) A pale yellow crystalline solid of the composition $2 \mathrm{c} \cdot 0.5 \mathrm{Et}_{2} \mathrm{O}$ was obtained, isolated by filtration and gently dried in vacuum. The crystals partially lose the co-crystallized $\mathrm{Et}_{2} \mathrm{O}$ upon prolonged drying. Yield $57 \%, 0.45 \mathrm{~g}$, pale yellow crystals. ${ }^{1} \mathrm{H}$ NMR (200MHz, [D 8 THF): $\delta_{\mathrm{H}} 1.10\left(1 \mathrm{H}, \mathrm{dd},{ }^{2} J_{\mathrm{HP}} \sim 10 \mathrm{~Hz},{ }^{2} J_{\mathrm{HP}}=1.3 \mathrm{~Hz}, \mathrm{P}-\mathrm{CH}=\mathrm{P}\right), 1.12(3 \mathrm{H}, \mathrm{t}$, $\left.{ }^{3} J_{\mathrm{HH}}=7.0 \mathrm{~Hz}, \mathrm{CH}_{3} \mathrm{Et}_{2} \mathrm{O}\right), 1.15\left(6 \mathrm{H}, \mathrm{dd},{ }^{3} J_{\mathrm{HP}}=16.9 \mathrm{~Hz},{ }^{3} J_{\mathrm{HH}}=7.0 \mathrm{~Hz}, \mathrm{CH}_{3} i \mathrm{Pr}\right), 2.96(1 \mathrm{H}$, ddsept, $\left.{ }^{2} J_{\mathrm{HP}}=10.2 \mathrm{~Hz},{ }^{4} J_{\mathrm{HP}}=0.8 \mathrm{~Hz},{ }^{3} J_{\mathrm{HH}}=7.0 \mathrm{~Hz}, \mathrm{P}-\mathrm{CH} i \operatorname{Pr}\right), 3.39\left(2 \mathrm{H}, \mathrm{q},{ }^{3} J_{\mathrm{HH}}=7.0 \mathrm{~Hz}, \mathrm{CH}_{2}\right.$ $\mathrm{Et}_{2} \mathrm{O}$ ), 7.00-7.25 (6H, m, CH Ph ), 7.30-7.47 (6H, m, CH Ph), 7.47-7.62 (4H, m, CH Ph), 7.74$7.92(4 \mathrm{H}, \mathrm{m}, \mathrm{CH} \mathrm{Ph}) .{ }^{13} \mathrm{C}\{\mathrm{H}\} \mathrm{NMR}\left(100.6 \mathrm{MHz},\left[\mathrm{D}_{8}\right] \mathrm{THF}\right): \delta_{\mathrm{C}} 0.0\left(1 \mathrm{C}, \mathrm{dd},{ }^{1} J_{\mathrm{CP}}=116.2 \mathrm{~Hz}\right.$, $\left.{ }^{1} J_{\mathrm{CP}}=10.7 \mathrm{~Hz}, \mathrm{P}-\mathrm{CH}=\mathrm{P}\right), 15.5\left(1 \mathrm{C}, \mathrm{s}, \mathrm{CH}_{3} \mathrm{Et}_{2} \mathrm{O}\right), 16.7\left(2 \mathrm{C}, \mathrm{d},{ }^{2} J_{\mathrm{CP}}=2.7 \mathrm{~Hz}, \mathrm{CH}_{3} i \mathrm{Pr}\right), 26.5(1 \mathrm{C}$, $\left.\mathrm{dd},{ }^{1} J_{\mathrm{CP}} \sim 60 \mathrm{~Hz},{ }^{3} J_{\mathrm{CP}}=1.8 \mathrm{~Hz}, \mathrm{CH} i \mathrm{Pr}\right), 66.2\left(1 \mathrm{C}, \mathrm{s}, \mathrm{CH}_{2} \mathrm{Et}_{2} \mathrm{O}\right), 126.7$ (2C, s, $\left.p-\mathrm{CH} \mathrm{Ph}\right), 128.0$ $\left(4 \mathrm{C}, \mathrm{d}, J_{\mathrm{CP}}=6.1 \mathrm{~Hz}, \mathrm{CH} \mathrm{Ph}\right), 129.0\left(4 \mathrm{C}, \mathrm{d}, J_{\mathrm{CP}}=10.8 \mathrm{~Hz}, \mathrm{CH} \mathrm{Ph}\right), 131.6\left(2 \mathrm{C}, \mathrm{d},{ }^{4} J_{\mathrm{CP}}=2.6 \mathrm{~Hz}\right.$, $p$-CH Ph), $132.3\left(4 \mathrm{C}, \mathrm{d}, J_{\mathrm{CP}}=18.7 \mathrm{~Hz}, \mathrm{CH} \mathrm{Ph}\right), 132.5\left(2 \mathrm{C}, \mathrm{dd},{ }^{1} J_{\mathrm{CP}} \sim 80 \mathrm{~Hz},{ }^{3} J_{\mathrm{CP}}=3.2 \mathrm{~Hz}, i-\mathrm{C}\right.$ $\mathrm{Ph}), 133.3\left(4 \mathrm{C}, \mathrm{dd},{ }^{2} J_{\mathrm{CP}}=8.4 \mathrm{~Hz},{ }^{4} J_{\mathrm{CP}}=1.9 \mathrm{~Hz}, o-\mathrm{CH} \mathrm{Ph}\right), 148.9\left(2 \mathrm{C}, \mathrm{dd},{ }^{1} J_{\mathrm{CP}}=12.2 \mathrm{~Hz},{ }^{3} J_{\mathrm{CP}}=\right.$ $9.4 \mathrm{~Hz}, i-\mathrm{C} \mathrm{Ph}) .{ }^{31} \mathrm{P}\{\mathrm{H}\} \mathrm{NMR}\left(81 \mathrm{MHz},\left[\mathrm{D}_{8}\right] \mathrm{THF}\right) \delta_{\mathrm{P}}-15.7\left(1 \mathrm{P}, \mathrm{d},{ }^{2} J_{\mathrm{PP}}=135.7 \mathrm{~Hz}\right), 33.7(1 \mathrm{P}, \mathrm{d}$, ${ }^{2} J_{\mathrm{PP}}=135.7 \mathrm{~Hz}$ ). Anal. Calcd for $\mathrm{C}_{30} \mathrm{H}_{33} \mathrm{P}_{2} \mathrm{O}_{0.5}$ (463.54): C, 77.73; H, 7.18\%. Found: C, 77.49; $\mathrm{H}, 7.28 \%$. Suitable crystals of $\mathbf{2 c} \cdot 0.5 \mathrm{Et}_{2} \mathrm{O}$ for $\mathrm{X}$-ray diffraction experiments were obtained directly from the reaction mixture. 


\section{Synthesis of [(diphenylphosphino)methylene]diphenyl[(2,4,6-trimethylphenyl)methyl]-}

phosphorane (2d). A solution of $\mathrm{KO} t \mathrm{Bu}$ in THF $(7.7 \mathrm{ml}, 1 \mathrm{M})$ was added to a stirred suspension of $1 \mathbf{d} \cdot(4.61 \mathrm{~g}, 7.71 \mathrm{mmol})$ in THF $(40 \mathrm{ml})$ at ambient temperature. The resulting mixture was stirred for an additional hour and filtered over diatomaceous earth to remove precipitated $\mathrm{KBr}$. The obtained yellow solution was reduced to dryness and dried in vacuum. The resulting solid foam was taken up in diethyl ether $(40 \mathrm{ml})$. A small amount of formed solid $(\sim 0.4 \mathrm{~g})$ was removed by filtration and discarded. The yellow solution was reduced to dryness, leaving an offwhite solid. This residue was suspended in heptane $(20 \mathrm{ml})$, isolated by filtration and dried in vacuum.

2d. Pale yellow solid, yield $82 \%, 3.27 \mathrm{~g}$, mp 138-140 ${ }^{\circ} \mathrm{C} .{ }^{1} \mathrm{H}$ NMR $\left(200 \mathrm{MHz},\left[\mathrm{D}_{8}\right] \mathrm{THF}\right): \delta_{\mathrm{H}} 1.06$ $\left(1 \mathrm{H}, \mathrm{dd},{ }^{2} J_{\mathrm{HP}}=11.2 \mathrm{~Hz},{ }^{2} J_{\mathrm{HP}}=2.7 \mathrm{~Hz}, \mathrm{P}-\mathrm{CH}=\mathrm{P}\right), 1.87\left(6 \mathrm{H}, \mathrm{s}, o-\mathrm{CH}_{3} \mathrm{Mes}\right), 2.20\left(3 \mathrm{H}, \mathrm{d}, J_{\mathrm{HP}}=2.1\right.$ $\left.\mathrm{Hz}, p-\mathrm{CH}_{3} \mathrm{Mes}\right), 4.11\left(2 \mathrm{H}, \mathrm{d},{ }^{2} J_{\mathrm{HP}}=14.7 \mathrm{~Hz}, \mathrm{P}-\mathrm{CH}_{2}\right), 6.68(2 \mathrm{H}, \mathrm{s}, \mathrm{CH} \mathrm{Mes}), 7.04-7.34(10 \mathrm{H}, \mathrm{m}$, $\mathrm{CH} \mathrm{Ph}$ ), 7.37-7.58 (10H, m, CH Ph). ${ }^{13} \mathrm{C}\{\mathrm{H}\}$ NMR (100.6 MHz, [D $]$ THF): $\delta_{\mathrm{C}} 10.5$ (1C, dd, $\left.{ }^{1} J_{\mathrm{CP}}=117.0 \mathrm{~Hz},{ }^{1} J_{\mathrm{CP}}=10.3 \mathrm{~Hz}, \mathrm{P}-\mathrm{CH}=\mathrm{P}\right), 20.8\left(1 \mathrm{C}, \mathrm{s}, p-\mathrm{CH}_{3} \mathrm{Mes}\right), 21.3\left(2 \mathrm{C}, \mathrm{s}, o-\mathrm{CH}_{3} \mathrm{Mes}\right)$, $32.2\left(1 \mathrm{C}, \mathrm{dd},{ }^{1} J_{\mathrm{CP}}=57.5 \mathrm{~Hz},{ }^{3} J_{\mathrm{CP}}=12.1 \mathrm{~Hz}, \mathrm{P}-\mathrm{CH}_{2}\right), 126.8(2 \mathrm{C}, \mathrm{s}, p-\mathrm{CH} \mathrm{Ph}), 128.0\left(4 \mathrm{C}, \mathrm{d}, J_{\mathrm{CP}}=\right.$ $6.2 \mathrm{~Hz}, \mathrm{CH} \mathrm{Ph}), 128.5\left(4 \mathrm{C}, \mathrm{d}, J_{\mathrm{CP}}=11.2 \mathrm{~Hz}, \mathrm{CH} \mathrm{Ph}\right), 128.8\left(1 \mathrm{C}, \mathrm{d},{ }^{2} J_{\mathrm{CP}}=8.0 \mathrm{~Hz}, i-\mathrm{C} \mathrm{Mes}\right)$, $129.6\left(2 \mathrm{C}, \mathrm{d},{ }^{4} J_{\mathrm{CP}}=2.7 \mathrm{~Hz}, m-\mathrm{CH} \mathrm{Mes}\right), 131.7\left(2 \mathrm{C}, \mathrm{d},{ }^{4} J_{\mathrm{CP}}=2.3 \mathrm{~Hz}, p-\mathrm{CH} \mathrm{Ph}\right), 132.5(4 \mathrm{C}, \mathrm{d}$, $\left.J_{\mathrm{CP}}=19.0 \mathrm{~Hz}, \mathrm{CH} \mathrm{Ph}\right), 133.6\left(2 \mathrm{C}, \mathrm{dd},{ }^{1} J_{\mathrm{CP}} \sim 80 \mathrm{~Hz},{ }^{3} J_{\mathrm{CP}}=1.5 \mathrm{~Hz}, i-\mathrm{C} \mathrm{Ph}\right), 133.9\left(4 \mathrm{C}, \mathrm{d}, J_{\mathrm{CP}}=\right.$ $9.2 \mathrm{~Hz}, \mathrm{CH} \mathrm{Ph}), 136.6\left(1 \mathrm{C}, \mathrm{d},{ }^{5} J_{\mathrm{CP}}=3.6 \mathrm{~Hz}, p-\mathrm{C} \mathrm{Mes}\right), 138.4\left(2 \mathrm{C}, \mathrm{d},{ }^{3} J_{\mathrm{CP}}=4.6 \mathrm{~Hz}, o-\mathrm{C} \mathrm{Mes}\right)$, $148.6\left(2 \mathrm{C}\right.$, pseudo-t, $\left.{ }^{1} J_{\mathrm{CP}}={ }^{3} J_{\mathrm{CP}}=10.5 \mathrm{~Hz}, i-\mathrm{C} \mathrm{Ph}\right) .{ }^{31} \mathrm{P}\{\mathrm{H}\} \mathrm{NMR}\left(81 \mathrm{MHz},\left[\mathrm{D}_{8}\right] \mathrm{THF}\right) \delta_{\mathrm{P}}-15.8$ $\left(1 \mathrm{P}, \mathrm{d},{ }^{2} J_{\mathrm{PP}}=150.6 \mathrm{~Hz}\right), 24.2\left(1 \mathrm{P}, \mathrm{d},{ }^{2} J_{\mathrm{PP}}=150.6 \mathrm{~Hz}\right)$. Anal. Calcd for $\mathrm{C}_{35} \mathrm{H}_{34} \mathrm{P}_{2}$ (516.60): C, 81.37 H, 6.63\%. Found: C, 81.25; H, 6.76\%. Suitable crystals of 2d for X-ray diffraction experiments were obtained by cooling a saturated solution in $\mathrm{Et}_{2} \mathrm{O}$ from ambient temperature to $-20{ }^{\circ} \mathrm{C}$.

\section{Synthesis of lithium-[(diphenylphosphino)methylene](methylene)diphenylphosphorane (4).}

A solution of $n$-butyllithium in hexane $(0.72 \mathrm{ml}, 1.6 \mathrm{M})$ was added to a stirred yellowish solution of $2 \mathrm{a} \cdot(0.46 \mathrm{~g}, 1.15 \mathrm{mmol})$ in toluene $(15 \mathrm{ml})$ at ambient temperature. The initially clear reaction mixture was stirred for an additional hour resulting in precipitation of a white solid. Afterwards, the formed product was collected by filtration, washed with cold toluene $(5 \mathrm{ml})$ and dried in vacuum.

4.0.5 toluene. Off-white solid, yield $67 \%, 0.35$ g. ${ }^{1} \mathrm{H}$ NMR $\left(200 \mathrm{MHz},\left[\mathrm{D}_{8}\right] \mathrm{THF}\right): \delta_{\mathrm{H}}-0.29(2 \mathrm{H}$, $\left.\mathrm{d},{ }^{2} J_{\mathrm{HP}}=9.3 \mathrm{~Hz}, \mathrm{Li}-\mathrm{CH}_{2}-\mathrm{P}\right), 1.12\left(1 \mathrm{H}, \mathrm{dd},{ }^{2} J_{\mathrm{HP}}=11.0 \mathrm{~Hz},{ }^{2} J_{\mathrm{HP}}=8.9 \mathrm{~Hz}, \mathrm{P}-\mathrm{CH}=\mathrm{P}\right), 2.32(1.5 \mathrm{H}, \mathrm{s}$, $\mathrm{CH}_{3}$ toluene), 7.0-7.3 (14.5, m, $\mathrm{CH} \mathrm{Ph}+$ toluene), 7.5-7.8 (8H, m, CH Ph). ${ }^{13} \mathrm{C}\{\mathrm{H}\} \mathrm{NMR}(100.6$ $\left.\mathrm{MHz},\left[\mathrm{D}_{8}\right] \mathrm{THF}\right): \delta_{\mathrm{C}}-2.1\left(1 \mathrm{C}, \mathrm{br}, \mathrm{Li}-\mathrm{CH}_{2}-\mathrm{P}\right), 14.5\left(1 \mathrm{C}, \mathrm{dd},{ }^{1} J_{\mathrm{CP}}=135.0 \mathrm{~Hz},{ }^{1} J_{\mathrm{CP}}=19.2 \mathrm{~Hz}, \mathrm{P}-\right.$ $\mathrm{CH}=\mathrm{P}), 21.4$ (0.5C, s, $\mathrm{CH}_{3}$ toluene), 125.9 (0.5C, s, $p$-CH toluene), 126.4 (2C, s, $p$ - $\mathrm{CH} \mathrm{Ph),}$ $127.7\left(4 \mathrm{C}, \mathrm{d}, J_{\mathrm{CP}} \sim 10 \mathrm{~Hz}, \mathrm{CH} \mathrm{Ph}\right), 127.8$ (4C, d, $\left.J_{\mathrm{CP}} \sim 7 \mathrm{~Hz}, \mathrm{CH} \mathrm{Ph}\right), 128.3\left(2 \mathrm{C}, \mathrm{d},{ }^{4} J_{\mathrm{CP}}=1.8\right.$ $\mathrm{Hz}, p-\mathrm{CH} \mathrm{Ph}), 128.8$ (1C, s, $m-\mathrm{CH}$ toluene), 129.6 (1C, s, o-CH toluene), 131.3 (4C, d, $J_{\mathrm{CP}}=9.2$ $\mathrm{Hz}, \mathrm{CH} \mathrm{Ph}), 132.3$ (4C, d, $\left.J_{\mathrm{CP}}=16.2 \mathrm{~Hz}, \mathrm{CH} \mathrm{Ph}\right), 138.3$ (0.5C, s, i-C toluene), 145.9 (2C, d, ${ }^{1} J_{\mathrm{CP}}$ $=67.3 \mathrm{~Hz}, i-\mathrm{C} \mathrm{Ph}), 148.8\left(2 \mathrm{C}, \mathrm{d},{ }^{1} J_{\mathrm{CP}}=8.3 \mathrm{~Hz}, i-\mathrm{C} \mathrm{Ph}\right) .{ }^{31} \mathrm{P}\{\mathrm{H}\} \mathrm{NMR}\left(81 \mathrm{MHz},\left[\mathrm{D}_{8}\right] \mathrm{THF}\right): \delta_{\mathrm{P}}-$ 
$13.4\left(1 \mathrm{P}, \mathrm{dq},{ }^{2} J_{\mathrm{PP}}=140.1 \mathrm{~Hz},{ }^{1} J_{\mathrm{LiP}}=36.5 \mathrm{~Hz}\right), 36.4\left(1 \mathrm{P}, \mathrm{d},{ }^{2} J_{\mathrm{PP}}=139.5 \mathrm{~Hz}\right) .{ }^{7} \mathrm{Li}\{\mathrm{H}\} \mathrm{NMR}$ $\left(155.5 \mathrm{MHz},\left[\mathrm{D}_{8}\right] \mathrm{THF}\right): \delta_{\mathrm{Li}} 0.09\left(\mathrm{~d},{ }^{1} J_{\mathrm{LiP}}=35.3 \mathrm{~Hz}\right)$.

A small portion of $\mathbf{4} \cdot 0.5$ toluene was recrystallized from $N, N, N^{\prime}, N^{\prime}$-tetramethylethylendiamine (tmeda) to obtain suitable crystals of the formula [(tmeda) $\left.\mathrm{Li}\left(\mathrm{Ph}_{2} \mathrm{PCHPPh}_{2} \mathrm{CH}_{2}\right\}\right] \cdot 0.5$ tmeda (4a) for X-ray diffraction experiments.

Supporting Information available. Crystal data and refinement details of 1a-4a, molecular structures and numbering schemes of $\mathbf{1 a}, \mathbf{1 b}, \mathbf{1 e}, \mathbf{2 a}, \mathbf{2 b}$, and $\mathbf{3}$. Additionally, crystallographic data (excluding structure factors) has been deposited with the Cambridge Crystallographic Data Centre as supplementary publication CCDC-842321 for $\mathbf{1 a} \cdot 2 \mathrm{CHCl}_{3}, \mathrm{CCDC}-842322$ for $\mathbf{1 b} \cdot 0.5$ toluene, CCDC-847440 for $\mathbf{1 b} \cdot$ toluene, CCDC-842323 for $\mathbf{1 c} \cdot \mathrm{CHCl}_{3}, \mathrm{CCDC}-842324$ for 1d, CCDC-856224 for 1e $2 \mathrm{CH}_{2} \mathrm{Cl}_{2}, \mathrm{CCDC}-842325$ for 3, CCDC-842326 for 2a, CCDC-842327 for $\mathbf{2 b}, \mathrm{CCDC}-842328$ for $\mathbf{2 c} \cdot 0.5 \mathrm{Et}_{2} \mathrm{O}, \mathrm{CCDC}-842329$ for $\mathbf{2 d}$, and CCDC-842330 for $4 \mathbf{a} \cdot 0.5$ tmeda. Copies of the data can be obtained free of charge on application to CCDC, 12 Union Road, Cambridge CB2 1EZ, UK [E- mail: deposit@ccdc.cam.ac.uk].

\section{References}

1. (a) Wittig, G.; Geissler, G. Liebigs Ann. Chem. 1953, 580, 44-57. (b) Wittig, G.; Schöllkopf, U. Chem. Ber. 1954, 87, 1318-1330. (c) Wittig, G., Haag, W. Chem. Ber. 1955, 88, 16541666.

2. (a) Edmonds, M.; Abell, A. In Modern Carbonyl Olefination: Methods and Applications; Takeda, T. Ed.; Wiley-VCH: Weinheim, 2004; pp 1-16. (b) Carey, F. A.; Sundberg, R. J. Advanced Organic Chemistry: Part B: Reactions and Synthesis, 5th Edn.; Springer: Berlin, Heidelberg, New York; 2007, pp 157-170.

3. Maryanoff, B. E.; Reitz, A. B. Chem. Rev. 1989, 89, 863-927.

4. (a) Gilheany, D. G. Chem. Rev. 1994, 94, 1339-1374. (b) Noury S.; Silvi B. Inorg. Chem. 2002, 41, 2164-2172.

5. (a) Kaska, W. C. Coord. Chem. Rev. 1983, 48, 1-58. (b) Cristau, H.-J. Chem. Rev. 1994, 94, 1299-1313. (c) Kolodiazhnyi, O. I. Tetrahedron 1996, 52, 1855-1929.

6. (a) Oosawa, Y.; Miyamoto, T.; Saito, T.; Sasaki, Y. Chem. Lett. 1975, 33-34. (c) Oosawa, Y.; Urabe, H.; Saito, T.; Sasaki, Y. J. Organomet. Chem. 1976,122, 113-121. (b) Holy, N.; Deschler, U.; Schmidbaur, H. Chem. Ber. 1982, 115, 1379-1388.

7. For recent examples see: (a) Sabounchei, S. J.; Samiee, S.; Salehzadeh, S.; Nojini, Z. B.; Bayat, M.; Irran, E.; Borowski, M. Inorg. Chim. Acta 2010, 363, 3654-3661. (b) Sabounchei, S. J.; Samiee, S.; Nematollahi, D.; Naghipour, A.; Morales-Morales, D. Inorg. Chim. Acta 2010, 363, 3973-3980. (c) Sabounchei, S. J.; Samiee, S.; Salehzadeh, S.; Nojini, Z. B.; Irran, E. J. Organomet. Chem. 2010, 695, 1441-1450. (d) Ebrahim, M. M.; Panchanatheswaran, K.; Neels, A., Stoeckli-Evans, H. J. Organomet. Chem. 2009, 694, 643- 
648. (e) Ebrahim, M. M.; Stoeckli-Evans, H.; Panchanatheswaran, K. Polyhedron 2007, 26, 3491-3495. (f) Fernandez, S.; Navarro, R.; Urriolabeitia, E. P. J. Organomet. Chem. 2000, $602,151-157$.

8. (a) Langer, J.; Fabra, M. J.; García-Orduña, P.; Lahoz, F. J.; Görls, H.; Oro, L. A.; Westerhausen, M. Dalton Trans. 2010, 7813-7821. (b) Langer, J.; Wimmer, K.; Görls, H.; Westerhausen, M. Dalton Trans. 2009, 2951-2957. (c) Langer, J.; Fabra, M. J.; GarcíaOrduña, P.; Lahoz, F. J.; Oro, L. A. Chem. Commun., 2008, 4822-4824.

9. Issleib, K.; Abicht, H. P. J. Prakt. Chem. 1970, 312, 456-465.

10. (a) Schmidbaur, H.; Deschler, U. Chem. Ber. 1981, 114, 2491-2500. (b) Schmidbaur, H.; Deschler, U. Chem. Ber. 1983, 116, 1386-1392.

11. Stephenson, B.; Solladie, G.; Mosher, H. S. J. Am. Chem. Soc. 1972, 94, 4184-4188 and references therein.

12. (a) Wohlleben, A.; Schmidbaur H. Anqew. Chem. 1977, 89, 428-429; Angew. Chem. Int. Ed. Engl. 1977, 16, 417-418. (b) Schmidbaur, H.; Wohlleben-Hammer, A. Chem. Ber. 1979, 112, 510-516.

13. (a) Taillefer, M.; Cristau, H. J.; Fruchier, A.; Vicente, V. J. Organomet. Chem. 2001, 624, 307-315. (b) Kaddouri, H.; Vicente, V., Ouali,A.; Ouazzani, F.; Taillefer, M. Angew. Chem. Int. Ed. 2009, 48, 333-336.

14. Spannenberg, A.; Müller, B. H.; Rosenthal, U. Z. Kristallogr. NCS 2005, 220, 581-584.

15. Schmidbaur, H.; Deschler, U.; Milewski-Mahrla, B. Angew. Chem. 1981, 93, 598-599; Angew. Chem. Int. Ed. Engl. 1981, 20, 586-588.

16. (a) Avent, A. G.; Bonafoux, D.; Eaborn, C.; Hill, M. S.; Hitchcock, P. B.; Smith, J. D. J. Chem. Soc., Dalton Trans. 2000, 2183-2190. (b) Fryzuk, M. D.; Giesbrecht, G. R.; Rettig, S. J. Organometallics 1997, 16, 725-736. (c) Colquhoun, I. J.; McFarlane, H. C. E.; McFarlane, W. J. Chem. Soc., Chem. Commun. 1982, 220-221. 